\title{
FINITE DIMENSIONAL TEICHMÜLLER SPACES AND GENERALIZATIONS
}

\author{
BY LIPMAN BERS ${ }^{1}$
}

\author{
CONTENTS \\ 0. Background \\ 1. Introduction \\ 2. Teichmüller spaces and modular groups \\ 3. Teichmüller's theorem \\ 4. Real boundaries \\ 5. Classification of modular transformations \\ 6. Embedding into complex number space \\ 7. Metrics in Teichmüller space \\ 8. Quasi-Fuchsian groups \\ 9. Fiber spaces over Teichmüller spaces \\ 10. Complex boundaries \\ 11. $b$-groups \\ 12. Ahlfors' problem, Sullivan's theorem and the complex boundary \\ 13. The Maskit embedding \\ 14. Riemann surfaces with nodes \\ 15. Strong deformation spaces \\ 16. Complex structure of deformation spaces \\ 17. Moduli spaces
}

\section{APPENDIX}

18. Some auxiliary results.

19. Infinite dimensional Teichmüller spaces

References

\section{Background.}

(A) This paper is an expanded version of one read at the Poincare Symposium of the American Mathematical Society at Bloomington, Indiana, in April 1980. The subject belongs to the "higher" theory of Riemann surfaces, and some readers may not object to being reminded of the main facts in the "standard" theory.

A Riemann surface $S$ is a connected surface on which one can do complex function theory which is, locally, not distinguishable from ordinary complex function theory in a domain of the complex number plane $\mathbf{C}$. More precisely, it is required that $S$ be a connected Hausdorff space, that certain continuous complex valued functions on subdomains of $S$ be designated as holomorphic, and that the following propositions be valid. (i) For every point $P$ of $S$ there

Received by the editors December 15, 1980.

${ }^{1}$ This material is based upon work supported by the National Science Foundation under Grant No. MCS 78-27119.

1980 Mathematics Subject Classification. Primary 14H1 5, 30C60, 30F40, 32G15. 
is an open neighborhood $N$ and a holomorphic function $\zeta: N \rightarrow \mathrm{C}$ which is a homeomorphism of $N$ onto a domain in $\mathbf{C}$; such a $\zeta$ is called a local parameter defined in $N$. (ii) A function $f: D \rightarrow C$ defined in a domain $D \subset S$ is holomorphic if and only if for every local parameter defined in $N \subset D$, the function $f \circ \zeta^{-1}$ is holomorphic (in the ordinary sense) in $\zeta(N) \subset \mathbf{C}$.

It follows that $S$ is a surface (locally homeomorphic to $\mathbf{R}^{2}$ ), orientable and, which is not quite obvious, triangulable.

(B) The simplest examples of Riemann surfaces are the complex $z$-plane $\mathbf{C}$ ( $z$ is a local parameter defined everywhere), the Riemann sphere $\hat{\mathbf{C}}=$ $\mathbf{C} \cup\{\infty\}$ ( $z$ is a local parameter in $\mathbf{C}, \zeta=1 / z$, with $\zeta=0$ at $\infty$, is a local parameter in $\hat{\mathbf{C}}-\{0\})$ and every domain $D \subset \mathbf{C}$, in particular, the upper half-plane $U=\{z \mid \operatorname{Im} z>0\}$. More generally, every subdomain of a Riemann surface is a Riemann surface.

A bijective homeomorphism $h$ between two Riemann surfaces $S_{1}$ and $S_{2}$ is called conformal if $f$ and $f^{-1}$ take (germs of) holomorphic functions into (germs of) holomorphic functions. Conformal Riemann surfaces are identical qua Riemann surfaces. Note that on a Riemann surface one can measure angles (use any local parameter). Conformal maps are angle and orientation preserving homeomorphisms.

Nonconstant globally defined holomorphic functions exist on any noncompact Riemann surface. On every Riemann surface, compact or not, there exist globally defined nonconstant meromorphic functions, i.e. functions which are holomorphic except for isolated poles. (The proofs always involve some variant of the Dirichlet principle.)

The ring of holomorphic functions on a noncompact Riemann surface $S$, and the field of meromorphic functions on any Riemann surface $S$, each determine $S$ uniquely, up to a conformal mapping and a reflection. (A reflection takes a Riemann surface $S$ into its mirror image $\bar{S} ; S$ and $\bar{S}$ are identical surfaces but the local parameters on $\bar{S}$ are the conjugates of the local parameters on $S$.)

(C) In a certain sense the theory of Riemann surfaces goes back to Gauss who proved in 1822 that a sufficiently small piece on a sufficiently smooth oriented surface $\Sigma$ in Euclidean space can be mapped conformally, i.e. preserving angles and orientation, onto a plane domain. Therefore any such $\Sigma$ can be made into a Riemann surface by declaring a nonconstant continuous complex-valued function $g: \Delta \rightarrow \mathbf{C}$, defined on a domain $\Delta \subset \Sigma$, to be holomorphic if it is locally, except at isolated points, a conformal mapping.

Indeed, the embedding $\Sigma \hookrightarrow \mathbf{R}^{N}$ is irrelevant; all that matters is the existence of a positive definite Riemannian metric

$$
d s^{2}=E(x, y) d x^{2}+2 F(x, y) d x d y+G(x, y) d y^{2}
$$

(which, in case $\Sigma \hookrightarrow \mathbf{R}^{N}$ can be taken as induced by the metric in $\mathbf{R}^{N}$ ). A local parameter on a part of $\Sigma$ is a complex-valued function

$$
\zeta=u(x, y)+i v(x, y)
$$


such that

$$
\frac{\partial(u, v)}{\partial(x, y)}>0, \quad d s^{2}=\rho(x, y)\left(d x^{2}+d y^{2}\right) .
$$

The real-valued functions $u, v$ are called isothermal parameters.

Gauss proved the existence of isothermal parameters $u, v$ under the hypothesis that $E, F, G$ are real analytic functions of the local coordinates $(x, y)$. Later this condition has been considerably weakened; finally Morrey (1938) showed that it is enough to assume that $E, F, G$ are measurable and the ratio

$$
\frac{E+G}{\sqrt{E G-F^{2}}}
$$

is bounded.

It is not difficult to verify that every Riemann surface can be defined by putting a properly chosen Riemannian metric onto an orientable sufficiently smooth surface. It is surprising that one may require that this metric be complete (every geodesic can be continued arbitrarily far) and have constant Gaussian curvature $+1,0$, or -1 ; then the metric is determined uniquely (except for an arbitrary constant factor in the case of zero curvature). Except in a few cases the canonical curvature is -1 . Let us recall how one finds this canonical metric.

(D) A deep theorem (the uniformization theorem of Klein-Poincaré-Koebe, 1882-1907) asserts that every simply connected Riemann surface is conformal to one of three:

$$
\hat{\mathbf{C}}, \mathbf{C} \text { and } U \text {. }
$$

If $S$ is any Riemann surface, then the general theory of covering spaces (created just for this purpose) shows that $S$ may be identified with $\tilde{S} / G$ where $\tilde{S}$ is the universal covering space of $S$, and $G$ the covering group. Now $\tilde{S}$ is simply connected and can be made into a Riemann surface by lifting local parameters from $S$ to $\tilde{S}$. Then $\tilde{S}$ becomes one of the three surfaces $(0.1)$ and $G$ becomes a discrete and fixed point free group of conformal self-mappings of $\tilde{S}$, hence a discrete group of Möbius transformations

$$
z \mapsto \frac{a z+b}{c z+d}, \quad a d-b c=1
$$

of $\tilde{S}$ onto itself.

One sees easily that if $\tilde{S}=\hat{\mathbf{C}}, G$ can contain only the identity so that $S=\hat{\mathbf{C}}$. If $\tilde{S}=\mathbf{C}$, then $G$ can contain only Euclidean translations. One verifies that in that case either $G=1$ (the trivial group), or $G$ is generated by one translation (say by $z \mapsto z+1$ ) or by two (say by $z \mapsto z+1$ and $z \mapsto$ $z+\tau, \operatorname{Im} \tau>0$ ). In the first case $S$ is conformal to $\mathbf{C}$, in the second to $\mathrm{C}-\{0\}$, and in the third $S$ is homeomorphic to a torus.

In all other cases $\tilde{S}=U$ and $G$ is a discrete group of Möbius transformations (0.2) subject to the condition that $a, b, c, d$ are real. Such groups have been named Fuchsian by Poincaré. 
Now $\hat{\mathbf{C}}$ carries a complete metric of Gaussian curvature 1 obtained by mapping $\hat{\mathbf{C}}$ stereographically onto the sphere $x_{1}^{2}+x_{2}^{2}+x_{3}^{2}=1$, and $\mathbf{C}$ has the canonical complete metric $|d z|=\sqrt{d x^{2}+d y^{2}}$ of curvature 0 . This metric is inherited by the infinite cylinder ( $C$ identified under the group $z \mapsto z+n$, $n \in \mathbf{Z}$ ) and the torus ( $\mathbf{C}$ identified under $z \mapsto n+m \tau ; n, m \in \mathbf{Z}, \operatorname{Im} \tau>0$ ). Finally, $U$ has a complete metric of curvature $(-1)$, the Poincaré metric $|d z| y^{-1}$ which makes it into a model of the non-Euclidean plane, and every Riemann surface distinct from a sphere, a sphere with one or two punctures and a torus is of the form $S=U / G, G$ a fixed point free Fuchsian group, and carries a Poincaré metric inherited from $U$.

(E) If $G$ is any Fuchsian group, which need not be torsion free, i.e. may have fixed points in $U$, then $S=U / G$ is again, in a natural way, a Riemann surface. The (globally defined) holomorphic and meromorphic functions on $S$ can be identified with holomorphic and meromorphic functions on $U$ which are invariant (automorphic) with respect to $G$. Similarly, for every integer $q$ the holomorphic and meromorphic q-differentials on $S$ can be identified with holomorphic and meromorphic functions $\varphi$ on $U$ with $\varphi(z) d z^{q} G$-invariant, i.e. satisfying

$$
\varphi(g(z)) g^{\prime}(z)^{q}=\varphi(z), \quad g \in G
$$

(automorphic forms). (A special convention is needed at elliptic and parabolic fixed points.)

(F) Fuchsian groups are special cases of Kleinian groups (this name is also due to Poincaré). A Kleinian group $G$ is a discrete group of not necessarily real Möbius transformations (0.2) with the following property: the limit set $\Lambda$ of $G$, defined as the set of accumulation points of trajectories $\{g(z), g \in G\}$ is not all of $\hat{\mathbf{C}}$. If so, the open dense set $\Omega=\hat{\mathbf{C}}-\Lambda$ is called the region of discontinuity of $G$ and every component of $\Omega$ is called a component of $G$. The group acts properly discontinuously on $\Omega$ and the quotient $\Omega / G$ is, in a natural way, a disjoint union of Riemann surfaces.

While the theory of Kleinian groups goes back to Klein, Poincaré and Koebe (and even earlier, to Schottky) a flowering of this theory occurred during the past fifteen years. A landmark in this development was Ahlfors' finiteness theorem: if a Kleinian group $G$ is finitely generated, the quotient $\Omega / G$ has finitely many components, each component is a compact Riemann surface or obtained from one by removing finitely many points, and the natural projection $\Omega \rightarrow \Omega / G$ is ramified over at most finitely many points.

(G) A compact Riemann surface $S$ is, topologically, a sphere with $p>0$ handles, the number $p$ is called the genus of $S$. The holomorphic function on $S$ are, of course, constants (by the maximum principle). The meromorphic functions form a field of algebraic functions of one variable (over $\mathbf{C}$ ). This means that any two meromorphic functions, $z$ and $w$, on $S$ are connected by an irreducible polynomial equation with complex coefficients

$$
P(z, w)=\sum_{\nu=1}^{n} \sum_{\mu=1}^{m} a_{\nu \mu} z^{\nu} w^{\mu}=0,
$$


and that, if these two functions are chosen suitably, any third is a rational function of the two.

In the latter case one says that $S$ is the Riemann surface of the plane algebraic curve (0.3). This does not mean, of course, that $S$ is isomorphic to the set of points $(z, w)$ in $\mathbf{C}^{2}$ satisfying $(0.2)$ or even to the set of points $\left(t_{1}, t_{2}, t_{3}\right)$ in the complex projective plane $P_{2}$ satisfying the corresponding homogeneous equation

$$
\sum_{\nu=1}^{n} \sum_{\mu=1}^{m} a_{\nu \mu} t_{1}^{\nu} t_{2}^{\mu} t_{3}^{n+m-\nu-\mu}=0 .
$$

Indeed, the curve $(0.3)$ will in general have singularities. However, every compact Riemann surface is isomorphic to a nonsingular algebraic curve in complex projective 3 -space.

(H) The theory of compact Riemann surfaces and the (more general) theory of algebraic curves over $\mathbf{C}$ are very well developed and exceedingly rich. Closely connected with compact Riemann surfaces are those with finitely generated fundamental groups. Such a surface $S$ is obtained from a compact surface $\hat{S}$, of some genus $p$ by removing $r \geqslant 0$ disjoint continua. If $n$ of those are points and $m=r-n$ are nondegenerate continua, we say that $S$ has type $(p, n, m)$, type $(p, n)$ if $m=0$.

A Riemann surface $S$ of type $(p, n, m)$, with $m>0$, is said to have $m$ ideal boundary curves. Such an $S$ can be always doubled, i.e., represented as a subdomain of a surface $S^{d}$, of type $(2 p+m-1,2 n, 0)$, which admits an anticonformal (angle preserving and orientation reversing) involution $j$ which leaves precisely $m$ disjoint Jordan curves $C_{1}, \ldots, C_{m}$ fixed and such that $S^{d}-\left\{C_{1} \cup \cdots \cup C_{m}\right\}$ as two components, one of which is $S$. The curves $C_{1}, \ldots, C_{m}$ are the ideal boundary curves of $S$ and $S^{d}$ is its Schottky double. (Example: the double of $U$ is $\hat{\mathbf{C}}$.)

(I) The uniformization theorem implies that every compact Riemann surface of genus 0 is conformal to a sphere (i.e. to $\hat{\mathbf{C}}$ ). The conformal type of a compact surface of genus 1 depends on one complex parameter $\tau \in U$. Already Riemann computed that the conformal type of a compact Riemann surface of genus $p>1$ depends on $3 p-3$ complex parameters (moduli) and this implies that the number of complex parameters for the conformal type of a surface of type $(p, n)$ is $3 p-3+n$, provided that number is not negative. Similarly, the conformal type of a surface of type $(p, n, m)$ with $m>0$ depends on $6 p-6+2 n+3 m$ real parameters, provided this number is not negative.

The main aim of Teichmüller theory is to make the dependence of a Riemann surface (of finite type) on the complex or real moduli as explicit and as transparent as possible. (The theory for type $(p, n, m)$ with $m>0$ can be reduced, at least in principle, to that of type $(p, n)$ by using doubling.)

(J) One of Teichmüller's great contributions was to recognize that the problem becomes more accessible if one considers not only conformal mappings between Riemann surfaces, but also quasiconformal mappings. 
Let $S$ and $\hat{S}$ be two homeomorphic Riemann surfaces and $f: S \rightarrow \hat{S}$ an orientation preserving diffeomorphism. If $P$ is a point on $S, \hat{P}=f(P) \in \hat{S}$, $z=x+i y$ a local parameter on $S$ defined near $P$ and $\hat{z}=\hat{x}+i \hat{y}$ one on $\hat{S}$ defined near $\hat{P}$, then the map $\hat{z}=h(z)$ defined by $h=\hat{z} \circ f \circ z^{-1} \mid N(N$ a sufficiently small neighborhood of $P$ ) is called a local representer of $f$ at $P$.

The map $f$ is called $K$-quasiconformal if there is a constant $K \geqslant 1$ such that, for every representer, at every point of $S$,

$$
\left|\frac{\partial \hat{z}}{\partial \bar{z}}\right| \leqslant \frac{K-1}{K+1}\left|\frac{\partial \hat{z}}{\partial z}\right|
$$

or, in real terms,

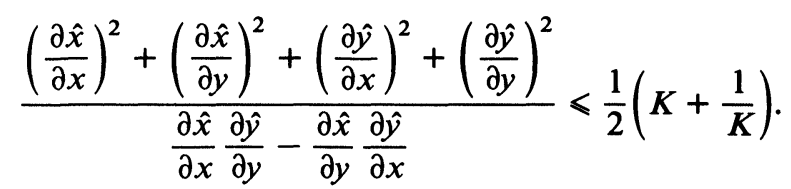

Geometrically this means that $f$ takes every infinitesimal circle into an infinitesimal ellipse whose major axis is at most $K$ times the minor axis.

The smallest $K$ for which $f$ is $K$-quasiconformal is called the dilatation of $f$ and is denoted by $K(f)$. If $K(f)=1, f$ is conformal.

The dilatation, or better, its logarithm, measures the deviation of $f$ from conformality and thus gives an upper bound for the difference between the conformal structures of $S$ and $\hat{S}$.

For technical reasons it is convenient not to insist that the orientation preserving homeomorphism $f: S \rightarrow \hat{S}$ be a diffeomorphism but only that the partial derivatives $\partial \hat{x} / \partial x, \partial \hat{y} / \partial x, \partial \hat{x} / \partial y, \partial \hat{y} / \partial y$ taken in the sense of distribution theory be locally square integrable measurable functions satisfying $\left(0.4^{\prime}\right)$ almost everywhere.

\section{Introduction.}

(A) Volume 1 of Acta Mathematica appeared in 1882; it begins with Poincarés paper on Fuchsian groups, the first of a series of memoirs which contain his work on discontinuous groups, automorphic functions and uniformization. Already on p. 6 Poincaré defines the Poincaré line element

$$
d s=\frac{|d z|}{y}
$$

in the upper half-plane $U=\{z=x+i y \mid y>0\}$ and explains, somewhat reluctantly, its geometric meaning: "Je ne puis passer sous silence le lien qui rattache les notions précédentes a la géométrie noneuclidienne de Lobatschewsky ... Cette terminologie m'a rendie de grands services de mes recherches, mais je ne l'employerai pas ici pour éviter toute confusion."

The memoir in question is concerned with constructing Fuchsian groups, i.e., discrete groups of plane non-Euclidean motions or, in the Poincaré half-plane model, discrete group of real Möbius transformations, i.e. discrete subgroups of $P S L(2, \mathbf{R})$. One such group, the elliptic modular group $\operatorname{PSL}(2, \mathbf{Z})$ was already well known at that time. For reasons which will become apparent later we shall denote this group by $\operatorname{Mod}_{1,1}$. 
(B) Now every point $\tau \in U$ defines a lattice $\mathcal{L}=\{\omega=n+m \tau \mid n, m \in \mathbf{Z}\}$ and a punctured torus $(\mathbf{C}-\mathcal{E}) / \mathcal{E}$ where we regard $\mathcal{E}$ as a subset and as an additive subgroup of $\mathbf{C}$. This torus is marked, i.e. it comes with a distinguished sequence of generators of the fundamental group, defined by the segments going from $z=0$ to $z=1$ and to $z=\tau$, respectively. Thus $U$ may be thought of as the space $T_{1,1}$ of isomorphism classes of marked Riemann surfaces of genus 1 with 1 puncture. Two distinct points $\tau_{1}$ and $\tau_{2}$ of $U$ represent conformally equivalent but differently marked Riemann surfaces if and only if $\tau_{2}=g\left(\tau_{1}\right)$ for some $g \in \operatorname{PSL}(2, \mathbf{Z})$.

Therefore $X_{1,1}=T_{1,1} / \operatorname{Mod}_{1,1}$ is the space of conformal equivalence classes (moduli) of once punctured Riemann surfaces of genus 1. Of course, $X_{1,1}$ is $\mathrm{C}$, the canonical mapping $T_{1,1} \rightarrow X_{1,1}$ being ramified over the points corresponding to $\tau=i$ and $\tau=(1+\sqrt{-3}) / 2$. The moduli space $T_{1,1}$ can be compactified by adding to it the point $\tau=\infty$ representing a thrice punctured sphere (obtained by 'degenerating' a punctured torus).

The part of Teichmüller space theory and an addition to this theory, on which we report here, deal with extending the statements made above to Riemann surfaces of genus $p$ with $n$ punctures, cf. $[5,14,26,30,35,66]$.

(C) We remark that the Teichmüller space $T_{p, n}$ is one of three distinct but related generalizations of the Poincare half-plane connected with the theory of Riemann surfaces. The other two are the Poincare model of the nonEuclidean 3-space:

$$
\mathbf{H}_{3}=\left\{(x, y, t) \in \mathbf{R}^{3} \mid t>0\right\}=\{(z, t) \mid t \in \mathbf{C}, t \in \mathbf{R}, t>0\}
$$

with the Poincaré metric

$$
d s^{2}=\frac{d x^{2}+d y^{2}+d t^{2}}{t^{2}}=\frac{|d z|^{2}+d t^{2}}{t^{2}},
$$

and the generalized Siegel upper half-plane $H_{p}$ consisting of complex symmetric $p \times p$ matrices $Z=X+i Y$ with positive definite imaginary parts, with the Siegel metric

$$
d s^{2}=\operatorname{trace}\left(Y^{-1}(d X+i d Y) Y^{-1}(d X-i d Y)\right) .
$$

(D) We assume acquaintance with the standard theory of Riemann surfaces, including uniformization theory and with the definition and basic properties of quasiconformal mappings in two dimensions, cf. $[13,15,66,81]$. We also assume the elementary properties of Kleinian groups [55].

In particular, we shall use without comment the theorem that a Riemann surface $S$, distinct from a sphere with 0,1 or 2 punctures and from a torus, can be represented as $U / G$ where $G$ is a torsion-free Fuchsian group determined by $S$ up to a conjugacy in $\operatorname{PSL}(2, \mathbf{R})$, and of the equivalent theorem that $S$ carries a uniquely determined Poincaré metric, i.e. a complete Riemannian metric of constant Gaussian curvature $(-1)$, which respects the conformal structure of $S$. The latter condition means that near every point of $S$ the metric can be written as $d s=\lambda(z)|d z|, z$ being a local parameter. 
The two equivalent statements just made constitute the main case of the so-called limit circle theorem the truth of which was perceived by Klein and by Poincaré in 1882, though a rigorous proof was given, by Poincaré and by Koebe, only in 1907.

(E) No claim of completeness is made for this report or for the bibliography. Analytic methods are emphasized throughout. The powerful geometric methods invented by Thurston $[54,117,118]$ could not be described here and only a few of his important results are mentioned. Important topics not covered also include the Grothendieck [62, 44, 53] and the Earle and Eells $[48,49]$ appoaches to Teichmüller theory, the Jenkins and Strebel quadratic differentials [69, 74, 107, 109], connections with the theory of Jacobians [47], and others [57, 74]. Teichmüller spaces of Fuchsian groups and infinite dimensional Teichmüller spaces are described briefly in the Appendix.

\section{Teichmüller spaces and modular groups.}

(A) Let $p$ and $n$ be nonnegative integers with $2 p-2+n>0$. In order to define $T_{p, n}$ precisely, we choose a topological oriented surface $\Sigma=\Sigma_{p, n}$ obtained by removing $n$ distinct points from a compact surface $\hat{\Sigma}$ of genus $p$ (with $\Sigma=\hat{\Sigma}$ if $n=0$ ) and call two orientation-preserving homeomorphisms of Riemann surfaces onto $\Sigma$

$$
f: S \rightarrow \Sigma \text { and } f^{\prime}: S^{\prime} \rightarrow \Sigma
$$

equivalent if there is a commutative diagram

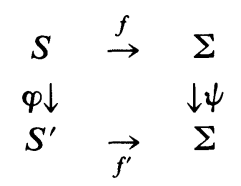

where $\varphi$ is an isomorphism (conformal mapping onto) and $\psi$ a homeomorphism homotopic to the identity. The equivalence classes

$$
[f]=[f: S \rightarrow \Sigma]
$$

of homeomorphisms of compact Riemann surfaces of genus $p$ with $n$ punctures onto $\Sigma$ are called marked Riemann surfaces of type $(p, n)$ or points of the Teichmüller space $T_{p, n}$.

(B) Every orientation-preserving homeomorphism $\omega$ of $\Sigma$ onto itself induces a mapping $\omega_{*}$ of $T_{p, n}$ onto itself, by the rule

$$
\omega_{*}([f])=[\omega \circ f] \text {. }
$$

Clearly $\omega_{*}$ depends only on the homotopy type of $\omega, \mathrm{id}_{*}=\mathrm{id}$ and $\left(\omega_{1} \circ \omega_{2}\right)_{*}$ $=\omega_{1 *}{ }^{\circ} \omega_{2 *}$. The transformations $\omega_{*}$ form the (Teichmüller) modular group $\operatorname{Mod}_{p, n}$.

Two points $\left[f_{1}\right]$ and $\left[f_{2}\right]$ of $T_{p, n}$ are equivalent under $\operatorname{Mod}_{p, n}$ if and only if the Riemann surfaces $f_{1}^{-1}(\Sigma)$ and $f_{2}^{-1}(\Sigma)$ are conformally equivalent. Therefore $X_{p, n}=T_{p, n} / \operatorname{Mod}_{p, n}$ is the space of moduli (conformal equivalence classes) of Riemann surfaces of type $(p, n)$. 
(C) There are several ways of topologizing $T_{p, n}$. They are all equivalent and lead to the following theorem.

The Teichmüller space $T_{p, n}$ is homeomorphic to $\mathbf{R}^{6 p-6+2 n}$ (and the modular

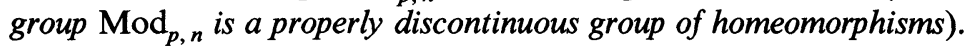

The first proof of this result is, in effect, due to Fricke [56], cf. [70].

(D) The topology in $T_{p, n}$ can be derived from the so-called Teichmüller metric. The Teichmüller distance between two points $\left[f_{1}\right]$ and $\left[f_{2}\right]$ of $T_{p, n}$ is defined as

$$
\left\langle\left[f_{1}\right],\left[f_{2}\right]\right\rangle=\frac{1}{2} \log \inf K(f)
$$

where $f$ ranges over all quasiconformal mappings in the homotopy class of $f_{1}^{-1} \circ f_{2}$, and $K(f)$ is the (global) dilatation of $f$.

If $f$ is a diffeomorphism, $K(f)$ is the supremum of the local dilatation $K(P ; f), P \in f_{2}^{-1}(\Sigma)$, where $K(P, f)$ is the ratio of the major axis to the minor axis of the infinitesimal ellipse into which $f$ maps an infinitesimal circle centered at $P$. For a general quasiconformal map the definition is the same, except that $K(P ; f)$ is defined only almost everywhere.

The Teichmüller space $T_{p, n}$ is a complete metric space, and the modular group $\operatorname{Mod}_{p, n}$ is a group of isometries (with respect to the Teichmüller metric).

(E) The space $T_{1,1}$ with the Teichmüler metric is isometric to $U$ with the Poincare metric; under this isometry $\operatorname{Mod}_{1,1}$ is the elliptic modular group.

The space $T_{0,3}$ is, of course, a point.

(F) Now let $\Sigma_{p, n, m}$ be a topological oriented surface obtained from a closed surface $\hat{\Sigma}$ of genus $p$ by removing $n+m$ distinct points which we divide into two sets consisting of $n$ and $m>0$ points, respectively. We assume that $6 p-6+2 n+3 m>0$.

The reduced Teichmüller space $T_{p, n, m}^{\#}$ consists of equivalence classes [ $f: S \rightarrow \Sigma$ ] of orientation-preserving homeomorphisms of Riemann surface of type $(p, n, m)$, i.e., of genus $p$, with $n$ punctures and $m$ ideal boundary curves, onto $\Sigma$. It is required that $f$ take the punctures of $S$ into the first set of punctures of $\Sigma$.

The space $T_{p, n, m}^{\#}$ is again a complete metric space (under the Teichmüller metric) and is homeomorpic to $\mathbf{R}^{6 p-6+2 n+3 m}$.

The definition of the (reduced) modular group $\operatorname{Mod}_{p, n, m}^{\#}$ is obvious. It is again a group of isometries and it acts properly discontinuously.

\section{Teichmüller's theorem.}

(A) Given two marked Riemann surfaces of type $(p, n)$,

$$
\left[f_{2}: S_{1} \rightarrow \Sigma\right] \text { and }\left[f_{2}: S_{2} \rightarrow \Sigma\right]
$$

we want to find an extremal map in the homotopy class of $f_{2}^{-1} \circ f_{1}$, that is a map $f: S_{1} \rightarrow S_{2}$ homotopic to $f_{2}^{-1} \circ f_{1}$ and with the smallest possible dilatation, namely

$$
K(f)=e^{2\left\langle\left[f_{1}\right],\left[f_{2}\right]\right\rangle} .
$$

The existence of an extremal map follows easily from general compactness properties of quasiconformal maps. Teichmüller discovered that the extremal 
map is unique and of a very special form: if it is not conformal, which can happen only if $\left[f_{1}\right]=\left[f_{2}\right]$, the extremal $f$ is, in the neighborhoods of all but finitely many points, a conformal map followed by an affine map followed by another conformal map.

(B) More precisely, a homeomorphism $f: S_{1} \rightarrow S_{2}$ is called a Teichmüller map if there are holomorphic quadratic differentials, $q_{1}$ on $S_{1}$ and $q_{2}$ on $S_{2}$, with

$$
0<\iint_{S_{1}}\left|q_{1}\right|=\iint_{S_{2}}\left|q_{2}\right|<\infty
$$

such that for every point $P \in S_{1}$, with $q_{1} \neq 0$ at $P$, we have that $q_{2} \neq 0$ at $f(P)$ and near $P$ the map may be written as

$$
\int q_{2}^{1 / 2}=K^{1 / 2} \operatorname{Re} \int q_{1}^{1 / 2}+\sqrt{-1} K^{-1 / 2} \operatorname{Im} \int q_{1}^{1 / 2}
$$

(for a proper choice of the signs of the square roots) where $K>1$ is constant, and is the dilatation of $f$. We call $q_{1}$ and $q_{2}$ the initial and terminal differentials of $f$.

(We recall that a holomorphic quadratic differential $q$ on a Riemann surface $S$ is a holomorphic section of twice the canonical bundle; locally, in terms of a local parameter $\zeta$ we have $q=\varphi(\zeta) d \zeta^{2}$ where $\varphi(\zeta)$ is a holomorphic function. Note that $|\varphi(\zeta)| d \xi d \eta$ is a density, so that condition (3.1) is meaningful. It implies that the singularities of $q_{j}$ at the punctures of $S_{j}$ are at worst simple poles.)

Relation (3.2) may be written as

$$
\xi_{2}=K^{1 / 2} \xi_{1}, \quad \eta_{2}=K^{-1 / 2} \eta_{1}
$$

where $\zeta_{1}=\xi_{1}+\sqrt{-1} \eta_{1}$ and $\zeta_{2}=\xi_{2}+\sqrt{-1} \eta_{2}$ are local parameters on $S_{1}$ and $S_{2}$, respectively, such that $\zeta_{1}=0$ at $P, \zeta_{2}=0$ at $f(P)$ and, near these points, $q_{1}=d \zeta_{1}^{2}, q_{2}=d \zeta_{2}^{2}$.

(C) The inverse $f^{-1}$ of a Teichmüller map is again a Teichmüller map, with the same dilatation $K$, with initial differential $\left(-q_{2}\right)$ and with terminal differential $\left(-q_{1}\right)$.

(D) Teichmüller's theorem asserts that $(\alpha)$ every Teichmüller map is the unique extremal in its homotopy class and $(\beta)$ that every extremal map is either conformal or a Teichmüller map.

Teichmüller first proved, in his famous 1939 paper [114], statement $(\alpha)$; in 1943 he derived $(\beta)$ from $(\alpha)$ by a continuity argument [115]. But his eccentric style (and unsavory political reputation) delayed the acceptance of his bold ideas until Ahlfors' paper [6] which contained a new proof of $(\beta)$. Later a modernized version of Teichmüller's own proof was given by the author [17], and a variational proof of $(\beta)$ was achieved by Schiffer [106] (in a special case), Krushkal [79, 80] and Hamilton [65].

(E) Kravetz [78] showed that through any two distinct points of $T_{p, n}$ there is a unique Teichmüller line, i.e. an isometric image of $\mathbf{R}$, and that three 
distinct points in $T_{p, n}$ are collinear if and only if they can be denoted by $\tau_{1}$, $\tau_{2}, \tau_{3}$ in such a way that $\left\langle\tau_{1}, \tau_{2}\right\rangle+\left\langle\tau_{2}, \tau_{3}\right\rangle=\left\langle\tau_{1}, \tau_{3}\right\rangle$.

At one time it was believed that the Teichmüller metric (which is not a Riemannian metric but can be derived from a Finsler metric (O'Byrne [97])) has negative curvature in the following sense: if $\tau_{0}, \tau_{1}, \tau_{1}^{\prime}, \tau_{2}$ and $\tau_{2}^{\prime}$ are points in Teichmüller space such that

$$
\left\langle\tau_{0}, \tau_{1}\right\rangle=\left\langle\tau_{1}, \tau_{2}\right\rangle=\frac{1}{2}\left\langle\tau_{0}, \tau_{2}\right\rangle, \quad\left\langle\tau_{0}, \tau_{1}^{\prime}\right\rangle=\left\langle\tau_{1}^{\prime}, \tau_{2}^{\prime}\right\rangle=\frac{1}{2}\left\langle\tau_{0}, \tau_{2}^{\prime}\right\rangle
$$

then

$$
\left\langle\tau_{2}, \tau_{2}^{\prime}\right\rangle>2\left\langle\tau_{1}, \tau_{1}^{\prime}\right\rangle \text {. }
$$

However, Masur [89] showed that this is not so if $\operatorname{dim} T_{p, n} \geqslant 2$.

Nevertheless, there are certain similarities between $T_{p, n}$ and spaces of negative curvature.

(F) The extremal problem considered in (A) can be generalized as follows. Let $f_{0}: S_{1} \rightarrow S_{2}$ be a given quasiconformal map between two Riemann surfaces, and if $S_{1}$ and $S_{2}$ have ideal boundary curves, let $\sigma$ be a closed set on the union of the ideal boundary curves of $S_{1}$. Consider all quasiconformal maps in the homotopy class of $f_{0}$ modulo $\sigma$, and find in it an extremal, i.e. one with the smallest possible dilatation.

(We recall that the quasiconformal map $f_{0}$ is defined, by continuity, also on the ideal boundary curves of $S_{1}$. Another quasiconformal map $f$ is homotopic to $f_{0}$ modulo $\sigma$ if $f_{0}^{-1} \circ f \mid \sigma=$ id and $f_{0}^{-1} \circ f$ can be continuously deformed into the identity keeping every point of $\sigma$ fixed.)

We assume, in order to exclude trivial cases, that the only conformal map $h: S_{1} \rightarrow S_{1}$ with $h \mid \sigma=$ id is the identity.

The existence of an extremal map follows again from compactness properties of quasiconformal maps.

A Teichmüller map $f: S_{1} \rightarrow S_{2}$ is defined as in (B), except that we now require that $q_{1}$ (and $q_{2}$ ) be continuous and real at all point of the ideal boundary curves of $S_{1}$ (of $S_{2}$ ) except at the points belonging to $\sigma\left(\right.$ to $f_{0}(\sigma)$ ).

If the fundamental group of $S_{1}$ is finitely generated and $\sigma$ is finite, Teichmüller's theorem (statements $(\alpha)$ and $(\beta)$ in (D)) is valid.

This can be proved, for instance, by doubling.

(G) Without any finiteness assumptions, statement $(\alpha)$ is always valid (Reich and Strebel [102, 103, 109, 112]). Also Hamilton [65] gave a necessary condition for an extremal map which, according to Reich and Strebel, is also sufficient.

On the other hand, statement $(\beta)$ is in general false. There are extremal maps which are not unique (Strebel [108]), and there are unique extremal maps which are not Teichmüller maps (in the sense defined above).

\section{Real boundaries.}

(A) Teichmüller's theorem gives a new proof of Fricke's result, see §2, (C). Indeed it yields a representation, or rather infinitely many representations, of $T_{p, n}$ as a ball in $\mathbf{R}^{6 p-6+2 n}$. 
We choose a point $\left[f_{0}: S_{0} \rightarrow \Sigma\right.$ ] as an origin of $T_{p, n}$ and we choose on $S_{0}$ a real basis of integrable quadratic (holomorphic) differentials $q_{1}, \ldots, q_{6 p-6+2 n}$ (the number $6 p-6+2 n$ comes from the Riemann-Roch theorem). The points $\tau=[f: S \rightarrow \Sigma]$ of $T_{p, n}$ distinct from the origin are in a one-to-one correspondence with Teichmüller maps $f_{\tau}: S_{0} \rightarrow S, f_{\tau}$ being the extremal map in the homotopy class of $f \circ f_{0}^{-1}$. The initial quadratic differential of $f_{\tau}$ can be written as

with

$$
\Lambda\left(\alpha_{1} q_{1}+\cdots+\alpha_{6 p-6+2 n} q_{6 p-6+2 n}\right)
$$

$$
\alpha_{1}^{2}+\cdots+\alpha_{6 p-6+n}^{2}=1, \quad \Lambda>0,
$$

and the dilatation $K$ of $f_{\tau}$ can be rewritten as

$$
K=(1+k) /(1-k), \quad 0<k<1 \text {. }
$$

We now assign to the origin in $T_{p, n}$ the origin in $\mathbf{R}^{6 p-6+n}$ and to the point $\tau$ the point in $\mathbf{R}^{6 p-6+2 n}$ with the coordinates $k \alpha_{1}, \ldots, k \alpha_{6 p-6+n}$. The resulting bijection of $T_{p, n}$ onto the open unit ball is a homeomorphism.

(B) This homeomorphism yields a compactification of $T_{p, n}$; the resulting boundary, called the Teichmüller boundary, is homeomorphic to the $(6 p-7$ $+2 n$ )-dimensional sphere and can be canonically identified with the set of rays in the space $Q\left(S_{0}\right)$ of integrable quadratic differentials on $S_{0}$.

It is natural to ask whether this compactification is essentially independent of the choice of the origin $\left[S_{0} \rightarrow \Sigma\right.$ ], and whether the action of $\operatorname{Mod}_{p, n}$ on $T_{p, n}$ extends continuously to the Teichmüller boundary. According to Kerckhoff [71] the answer to both questions is no.

(C) A compactification of $T_{p, n}$, which does not depend on the choice of an origin is due to Thurston ([117], cf. the presentation in [54]).

Let $\tau=[f: S \rightarrow \Sigma]$ be a point in $T_{p, n}, C$ a Jordan curve on $\Sigma$ homotopic neither to a point nor a puncture of $S$ and $l_{\tau}(C)$ the length of the unique Poincaré geodesic on $S$ freely homotopic to $f_{\tau}^{-1}(C)$. It is known that the knowledge of all numbers $l_{\tau}(C)$ determines $\tau$; as a matter of fact, it suffices to know these numbers for a properly chosen finite sequence $C_{1}, \ldots, C_{N}$. Also, since there are nonhomogeneous relations between the various numbers $l_{\tau}(C)$, it is enough to know these numbers up to a common positive factor.

Thus there is a bijection between $T_{p, n}$ and a subset $M$ of an infinitely dimensional (or, if one prefers, a finite dimensional) real projective space. This bijection is a homeomorphism and Thurston proves, in a highly imaginative way, that the closure $\bar{M}$ of $M$ is homeomorphic to a ball, while the Thurston boundary $\bar{M}-M$ is homeomorphic to a sphere, both of the "right" dimension.

For reasons which will become apparent later the Teichmüller and Thurston boundaries are called real boundaries.

The action of $\operatorname{Mod}_{p, n}$ extends continuously to the Thurston boundary (because it amounts to a permutation of the Jordan curves $C$ on $\Sigma$ ).

(D) In Thurston's proof $\bar{M}-M$ appears as the set of measured foliations on $\Sigma$, determined up to equivalence and up to a common positive factor. It is 
strange that just as the various Teichmüller boundaries, the Thurston boundary can be identified with the space of rays of $Q(S)$, for every $\tau=[S \rightarrow \Sigma]$ in $T_{p, n}$.

This follows from the fact, proved by Hubbard and Masur [68], and later by Kerckhoff [71], that every measured foliation on a Riemann surface is equivalent to one defined by a quadratic differential $q$, the leaves being the horizontal trajectories $q>0$, and the transverse measure being derived from the metric $d s=|q|^{1 / 2}$.

(E) The Teichmüller theorem stated in $\$ 2,(F)$ leads to a representation of $T_{p, n, m}^{\#}$ as a ball in $\mathbf{R}^{6 p-6+2 n+3 m}$. The argument is the same as in (A).

\section{Classification of modular transformations.}

(A) Thurston [117], cf. [54], used his compactification of Teichmüller space to classify orientation preserving homeomorphisms $\omega$ of a surface $\Sigma$ onto itself up to homotopy (or, which is the same, up to isotopy) and discovered that in the general case $\omega$ is isotopic to a mapping of a very special form. He himself indicated that these results could be obtained from those obtained by Nielsen many years ago; this was made explicit by J. Gilman [60] and by R. Miller [93]. In [31] the author showed how the classification can be obtained by a generalization of Teichmüller's extremal problem. We outline this approach below. (Further developments and applications may be found in Kra [75].)

(B) The elements $\gamma$ of $\operatorname{Mod}_{p, n}$ can be classified as follows: we call $\gamma \neq \mathrm{id}$ elliptic if it has a fixed point, parabolic if it has no fixed point but

$$
\inf \langle\tau, \gamma(\tau)\rangle=0 \quad\left(\tau \in T_{p, n}\right) .
$$

If $\gamma$ is neither elliptic nor parabolic, then

$$
a=\inf \langle\tau, \gamma(\tau)\rangle>0 \quad\left(\tau \in T_{p, n}\right)
$$

and $\gamma$ is called hyperbolic if there is a point $\tau_{0}$ with $\left\langle\tau_{0}, \gamma\left(\tau_{0}\right)\right\rangle=a$, pseudohyperbolic if not.

For $p=n=1$ this is the usual classification of real Möbius transformations (and the pseudohyperbolic case does not occur).

In all cases $\gamma$ is elliptic if and only if it is of finite order, and $\gamma$ is hyperbolic if and only if it leaves a Teichmüller line fixed.

(C) Every $\gamma \in \operatorname{Mod}_{p, n}$ is of the form $\gamma=\omega_{*}$ where $\omega$ is a topological orientation preserving self-mapping of $\Sigma$ (which may be chosen as a diffeomorphism if $\Sigma$ is given a differentiable structure) and the metric properties of $\omega_{*}$ reflect the topological properties of $\omega$. Thus $\omega_{*}$ is elliptic if and only if $\omega$ is homotopic to a periodic mapping.

A self-mapping $\omega: \Sigma \rightarrow \Sigma$ is called reduced by a finite nonempty set $C_{1}, \ldots, C_{k}$ of Jordan curves on $\Sigma$ such that the $C_{j}$ are disjoint, no $C_{j}$ is freely homotopic to a curve $C_{i}, i \neq j$, and no $C_{j}$ can be continuously deformed into a point or puncture of $\Sigma$, if

$$
\omega\left(C_{1} \cup \cdots \cup C_{k}\right)=C_{1} \cup \cdots \cup C_{k} .
$$


It turns out that $\gamma=\omega_{*}$ is hyperbolic if and only if $\omega$ is not homotopic to a periodic map and irreducible (not homotopic to a reduced map).

(D) A reducible map $\omega$ is always homotopic to a $\omega_{0}$ which is reduced by curves $C_{1}, \ldots, C_{k}$ and has the following property. For every component $\Pi$ of $\Sigma-\left(C_{1} \cup \cdots \cup C_{k}\right)$ set $\omega_{\Pi}=\omega_{0} \mid \Pi$ and let $r_{\Pi}$ denote the smallest positive integer such that $\omega_{\Pi}^{r_{\Pi}(\Pi)}=\Pi$. Then $g_{\Pi}=\omega_{\Pi}^{r_{\Pi}}$ is irreducible. It turns out that if $\omega$ is reducible and not homotopic to a periodic map then $\gamma=\omega_{*}$ is parabolic or pseudohyperbolic according to whether all or not all maps $g_{\Pi}$ are homotopic to periodic maps or the identity.

(E) If $\gamma=\omega_{*}$ is hyperbolic then, by definition, there are points $\tau=$ [f: $S \rightarrow \Sigma$ ] of $T_{p, n}$ with the following property: the self-map $f^{-1} \circ \omega \circ f$ of $S$ is homotopic to a Teichmüller map $g$ which is absolutely extremal, i.e. its dilatation cannot be decreased by changing $g$ within its homotopy class and by changing the conformal structure of $S$.

It can be shown that a Teichmüller map $g: S \rightarrow S$ is absolutely extremal if and only if its initial differential $q$ is also its terminal differential.

If so, $g$ is what Thurston calls a pseudo-Anosov diffeomorphism: there are two transversal measured foliations, the horizontal trajectories of $q$ and $(-q)$, respectively, such that $g$ maps them onto themselves while multiplying the distance between the leaves by $e^{2 a}$ and $e^{-2 a}$, respectively; here $a$ is the number defined by (5.2).

Thus a self-mapping $\omega: \Sigma \rightarrow \Sigma$ which is not homotopic to a periodic map is either homotopic to a pseudo-Anosov map or reducible, but not both, as has been first proved by Thurston.

(F) The elements of $\operatorname{Mod}_{p, n, m}^{\#}$ can be classified similarly. However, it turns out that if $m>0$, there are no hyperbolic elements.

\section{Embedding into complex number space.}

(A) Already Teichmüller guessed [116], and claimed to have proved, that the Teichmüller space $T_{p}=T_{p, 0}$ is, in a natural way, a complex manifold, but the first complete proof was given by Ahlfors in [7].

Note that there are certain functions on $T_{p}$ which must be holomorphic under a "natural" definition of the complex structure in $T_{p}$.

Choose a "canonical" integral homology basis $\alpha_{1}, \ldots, \alpha_{p}, \beta_{1}, \ldots, \beta_{p}$ on $\Sigma$, so that $\alpha_{i} \times \beta_{i}=-\left(\beta_{i} \times \alpha_{i}\right)=1, i=1, \ldots, p$, and "all" other intersection numbers vanish. For every $\tau=[f: S \rightarrow \Sigma] \in T_{p, 0}$ there are $p$ uniquely determined holomorphic Abelian differentials $a_{1}, \ldots, a_{p}$ on $S$ such that

$$
\int_{f^{-1}\left(\alpha_{j}\right)} a_{i}= \begin{cases}1 & \text { if } i=j \\ 0 & \text { if } i \neq j\end{cases}
$$

The $p \times p$ matrix $\Pi(\tau)$, with entries

$$
\Pi_{i j}(\tau)=\int_{f^{-1}\left(\beta_{j}\right)} a_{i}
$$


depends on $\tau$ only and is an element of the generalized Siegel upper half-plane $H_{p}($ see $\S 1,(\mathrm{C}))$. It is natural to require that the map $T_{p, 0} \rightarrow H_{p}$ just defined be holomorphic.

This requirement leads to a complex manifold structure on $T_{2}$ and on the part $T_{p}^{\prime}$ of $T_{p}, p>2$, corresponding to nonhyperelliptic surfaces; one can use as complex coordinates, near every point of $T_{2}$ or $T_{p}^{\prime}$, an appropriately chosen set of $3 p-3$ entries of the period matrix $\Pi$, as was observed by Rauch [100, 101]. The most delicate part of Ahlfors' argument consists in showing that the complex structure does not become singular on the set $T_{p}-T_{p}^{\prime}$.

(B) Actually there are in $T_{p, n}$ natural global complex coordinates: $T_{p, n}$, with its complex structure, can be realized as a bounded domain in complex number space [19].

Indeed, this realization is, to a certain extent, canonical; if we choose a point $\tau_{0}=\left[f_{0}: S_{0} \rightarrow \Sigma\right]$ as the "origin" in $T_{p, n}$, then $T_{p, n}$ can be represented canonically as a bounded domain in the $(3 p-3+n)$-dimensional complex vector space $Q\left(\bar{S}_{0}\right)$ of integrable quadratic differentials on the mirror image $\bar{S}_{0}$ of $S_{0}$. This is accomplished as follows.

(C) We represent $S_{0}$ as $U / G_{0}$ where $G_{0}$ is a torsion-free Fuchsian group (under our assumptions on $S_{0}, G_{0}$ is finitely generated and every real point is an accumulation point of an orbit). Note that $\bar{S}_{0}=L / G_{0}$ where $L$ is the lower half-plane.

The space $Q\left(\bar{S}_{0}\right)$ can be identified with the space $B_{2}\left(L, G_{0}\right)$ of holomorphic functions $\varphi(z), z \in L$, satisfying the functional equation of automorphic forms of weight $(-4)$

$$
\varphi(g(z)) g^{\prime}(z)^{2}=\varphi(z) \text { for } g \in G_{0}
$$

and the two equivalent growth conditions:

$$
\begin{gathered}
\iint_{L / G_{0}}|\varphi(z)| d x d y<\infty, \\
\sup \left|y^{2} \varphi(z)\right|<+\infty,
\end{gathered}
$$

which characterize the so-called cusp forms.

Now let $\tau=[f: S \rightarrow \Sigma]$ be any point of $T_{p, n}$. Without loss of generality we assume that $f_{0}$ and $f$ are quasiconformal, so is then the mapping $f^{-1} \circ f_{0}$. The Beltrami differential $m$ of $f^{-1} \circ f_{0}$ on $S_{0}$ can be lifted to a bounded (measurable) function $\mu(z)$ satisfying the inequality

$$
\|\mu\|_{\infty}=\operatorname{ess} \sup |\mu(z)|<1
$$

and the functional equation

$$
\mu(g(z)) \overline{\frac{g^{\prime}(z)}{g^{\prime}(z)}}=\mu(z), \quad g \in G_{0} .
$$

Let $w_{\mu}$ be the unique $\mu$-conformal mapping of $U$ onto itself which preserves the points $0,1, \infty$. (A univalent map $z \mapsto w(z)$ is called $\mu$-conformal if it satisfies the Beltrami equation 


$$
\frac{\partial w}{\partial \bar{z}}=\mu \frac{\partial w}{\partial z} \text {. }
$$

A $\mu$-conformal map of $U$ onto itself can be continued to a topological self-map of $U \cup \mathbf{R} \cup\{\infty\}$.)

One computes that for every $g \in G_{0}$ the map $w_{\mu} \circ g$ is again $\mu$-conformal, hence of the form $g_{\mu} \circ w_{\mu}$ with a holomorphic $g_{\mu}$, and since $g_{\mu}$ maps $U$ onto itself it is a real Möbius transformation. Thus $G_{\mu}=w_{\mu} G_{0} w_{\mu}^{-1}$ is again a torsion-free Fuchsian group, and one can show that there exists a commutative diagram

$$
\begin{array}{ccc}
U & \stackrel{w_{\mu}}{\rightarrow} & U \\
\downarrow & & \downarrow \\
S_{0}=U / G_{0} & \underset{f-1}{\rightarrow} & U / G_{\mu}=S
\end{array}
$$

where the vertical arrows represent canonical projection.

(D) One can prove that $w_{\mu} \mid \mathbf{R}$ as well as the isomorphism $g \mapsto w_{\mu} \circ g \circ w_{\mu}^{-1}$ of $G_{0}$ onto $G_{\mu}$ depend not on the choice of $f_{0}$ and $f$ but only on $\tau_{0}$ and $\tau$. On the other hand, if $\tau_{0}$ is given, any $\mu$ satisfying (6.4) and (6.5) defines a $\tau$ in $T_{p, n}$.

Now extend the definition of $\mu(z)$ to all of $\mathbf{C}$ by setting $\mu \mid L=0$. Let $w^{\mu}(z)$ denote the unique $\mu$-conformal map of $\mathbf{C}$ onto itself which leaves $0,1, \infty$ fixed. Then

$$
w_{\mu}=h_{\mu} \circ w^{\mu} \mid U
$$

where $h_{\mu}: w^{\mu}(U) \rightarrow U$ is conformal, and $w^{\mu} \mid L$ is also conformal. Furthermore, one can show that $w^{\mu} \mid L$ depends only on $\tau$ and $\tau_{0}$ and not on the particular choice of $\mu$. So does therefore the Schwarzian derivative

$$
\varphi^{\mu}(z)=\frac{d u}{d z}-\frac{1}{2} u^{2} \quad \text { where } u=\frac{d}{d z} \log \frac{d\left(w^{\mu} \mid L\right)}{d z} .
$$

One computes that $\varphi^{\mu}(z) d z^{2}$ is $G$-invariant, i.e., $\varphi^{\mu}$ satisfies (5.1). Also, by the Kraus-Nehari inequality for the Schwarzian derivatives of univalent functions [77, 96],

$$
\sup \left|y^{2} \varphi^{\mu}(z)\right| \leqslant \frac{3}{2}
$$

Thus $\varphi^{\mu} \in B_{2}(L, G)$.

(E) The mapping $\tau \mapsto \varphi^{\mu}$ of $T_{p, n}$ into $B_{2}\left(L, G_{0}\right)$ is continuous (this follows from the fact that $w^{\mu}(z)$ depends holomorphically on $\mu \in L_{\infty}(\mathbf{C})$ ). It is also injective. Indeed knowing $\varphi^{\mu}$ one can find $w^{\mu} \mid L$ as a quotient of two linearly independent solutions of the ordinary differential equation $2 \eta^{\prime \prime}=-\varphi^{\mu} \eta$. Knowing $w^{\mu} \mid L$ one can find $h_{\mu}$ as the conformal map of the Jordan domain complementary to $w^{\mu}(L)$ onto $U$ which keeps $0,1, \infty$ fixed, and knowing $h_{\mu}$ one obtains from (6.3) the function $w_{\mu} \mid \mathbf{R}$ which determines the isomorphism $G_{0} \rightarrow G_{\mu}$ and $\tau$. 
(F) We conclude that the mapping $\tau \mapsto \varphi^{\mu}$ is a homeomorphism of $T_{p, n}$ onto $a$ domain in $B_{2}\left(L, G_{0}\right) \approx \mathbf{C}^{3 p-3+n}$, a bounded domain, by (6.10). We shall often identify $T_{p, n}$ with its image.

One can either use this identification to define the complex structure in $T_{p, n}$ (then one must check that this structure does not depend on the choice of $\tau_{0}$ ) or, if this structure is already defined, one can verify that the map $\tau \mapsto \varphi^{\mu}$ is holomorphic.

(G) REMARK. It is easy to see that $T_{0,4}$ is isomorphic to $T_{1,1}, T_{0,5}$ to $T_{1,2}$ and $T_{0,6}$ to $T_{2,0}$. D. Patterson [98] showed that there are no other isomorphisms.

(H) The modular group $\operatorname{Mod}_{p, n}$ acts on $T_{p, n}$ as a group of holomorphic self-mapping. Since it also acts properly discontinuously, the moduli space

$$
X_{p, n}=T_{p, n} / \operatorname{Mod}_{p, n}
$$

is a normal complex space. It has singularities which come from the fixed points of $\operatorname{Mod}_{p, n}$ corresponding to Riemann surfaces admitting conformal self-mappings.

However, Grothendieck and Serre [62] observed that a subgroup of $\operatorname{Mod}_{p, n}$ of finite index operates freely, for instance the subgroup induced by selfmapping of $\Sigma$ which induce the identity on $H_{1}\left(\Sigma, \mathbf{Z}_{m}\right)$ where $m \geqslant 3$ is a fixed integer. Thus $X_{p, n}$ is a complex manifold divided by a finite group.

By methods of algebraic geometry Baily, Mumford and others $[16,40,95]$ showed that $X_{p, n}$ is a quasi-projective algebraic variety.

(I) Since the (complex) dimension of $T_{1,1}$ is 1 , this Teichmüller space is conformally equivalent to a disc. Numerical calculations show, however, that the image of $T_{1,1}$ in $B_{2}(L, G) \approx \mathrm{C}$ is not a disc (Porter [99]).

(J) In all cases $T_{p, n}$ is a domain of holomorphy. There are several proofs of this fact, the first (by Bers and Ehrenpreis [33]) is based on Ahlfors' characterization of quasi-circles, see $\S 18$, (C).

(K) On the other hand, if $\operatorname{dim} T_{p, n}>1, T_{p, n}$ is not a symmetric domain and even not homogeneous. This was proved by Royden [104, 105] who showed that, under the condition stated, the full group of holomorphic self-mappings of $T_{p, n}$ coincides with $\operatorname{Mod}_{p, n}$ or (in a few cases) contains it as a subgroup of finite index. Hence it is discrete.

(L) The space $T_{p, n, m}^{\#}$ has a natural real-analytic structure but, if $m>0$, no natural complex structure.

\section{Metrics in Teichmüller space.}

(A) Royden's result, stated in $\$ 6,(\mathrm{~K})$, is closely connected with another theorem also due to him.

The Teichmüller distance between two points $P$ and $Q$ in $T_{p, n}$ can be defined, without any reference to quasiconformal mappings, by the formula

$$
\langle P, Q\rangle=\inf \left\langle F^{-1}(P), F^{-1}(Q)\right\rangle
$$


where $\langle$,$\rangle on the right denotes the Poincare distance in U$ ( $=$ the Teichmüller distance in $T_{1,1}$ ) and $F$ runs over all holomorphic maps $U \rightarrow$ $T_{p, n}$. Royden's result can also be stated as follows: the Teichmüller metric coincides with the Kobayashi metric.

(The Kobayashi pseudo-metric [72] can be defined in every complex manifold; the general definition is more complicated than (7.1).)

(B) We mention at this occasion that given two distinct points $P$ and $Q$ in $T_{p, n}$ there is a holomorphic isometry $F: U \rightarrow T_{p, n}$ (an isometry with respect to the Poincaré metric in $U$ and the Teichmüller metric is $T_{p, n}$ ) such that $F(U)$ contains $P$ and $Q$ and hence also the Teichmüller line through these points. This was known already to Teichmüller.

Also, for every fixed $P \in T_{p, n}$, the distance $\langle P, Q\rangle$ is a differentiable function of $Q \neq P$ (Earle [46]).

(C) In every bounded domain $D$ in a complex number space one can define two metrics which are determined by the complex structure of $D$ and hence invariant under any holomorphic self-mapping of the domain. The Bergman metric is a Kähler metric defined by the line element

$$
d s^{2}=\Sigma \frac{\partial^{2} \log K(z ; z)}{\partial z_{i} \partial \bar{z}_{j}} d z_{i} \wedge d \bar{z}_{j}
$$

where $K(z ; \zeta)=K\left(z_{1}, \ldots, z_{\delta} ; \zeta_{1}, \ldots, \zeta_{\delta}\right)$ is the Bergman kernel of $D \subset \mathbf{C}^{\delta}$ The Carathéodory distance between two points $P$ and $Q$ of $D$ is defined as

$$
d_{\mathrm{Car}}(P, Q)=\sup \langle f(P), f(Q)\rangle,
$$

where $\langle$,$\rangle is again the Poincaré distance in U$ and $f$ runs over all holomorphic maps $D \rightarrow U$. It is not known whether the Carathéodory metric in $T_{p, n}$ coincides with the Teichmüller metric, but Earle [45] proved that it is complete; so is the Bergman metric since it always dominates the Carathéodory metric (Hahn [63]). The completeness of the Carathéodory metric implies again that $T_{p, n}$ is a domain of holomorphy. (This also follows from Royden's theorem.)

(D) Another Hermitian metric in $T_{p, n}$ invariant under $\operatorname{Mod}_{p, n}$, has been defined by Weil [120]. The tangent space to $T_{p, n}$ at a point $\tau=[f: S \rightarrow \Sigma]$ can be identified with $Q(\bar{S}) \approx B_{2}\left(L, G_{0}\right)$, as is seen from $\S 6$, and in $B_{2}\left(L, G_{0}\right)$ there is a canonical scalar product defined many years ago by Petersson:

$$
(\varphi, \psi)=\iint_{L / G} y^{2} \varphi(z) \overline{\psi(z)} d x d y .
$$

The Hermitian Weil-Petersson metric derived from this scalar product clearly has the required invariance. It has been shown to be Kählerian (Weil, Ahlfors [8, 9]) and to have negative Ricci and holomorphic sectional curvatures (Ahlfors). But it is incomplete (Chen [38], Wolpert [121]) and therefore distinct from the Bergman metric. (Cf. also $\$ 16,(\mathrm{E})$.) 


\section{Quasi-Fuchsian groups.}

(A) We return to the mapping $w^{\mu}$ and form the group

$$
G^{\tau}=w^{\mu} G_{0}\left(w^{\mu}\right)^{-1}
$$

It is ( $\alpha$ ) a group of not necessarily real Möbius transformations (this is established as the similar statement about $G_{\mu}$ in $\left.\$ 6,(C)\right),(\beta)$ it preserves a directed Jordan curve $C$ which is also its limit set, and set of accumulation points of orbits (namely the curve $\left.C=w^{\mu}(\mathbf{R} \cup\{\infty\})\right)$ and $(\gamma)$ it acts properly discontinuously on the two domains complementary to $C$ (namely $w^{\mu}(U)$ and $\left.w^{\mu}(L)\right)$. Actually $(\gamma)$ follows from $(\alpha)$ and $(\beta)$.

A group with the properties $(\alpha),(\beta),(\gamma)$ is called a quasi-Fuchsian group of the first kind (it is Fuchsian if $C$ is a circle or a line).

(B) Such a group always represents two Riemann surfaces, the quotients of the two Jordan domains by the group, and if the group is finitely generated, the two are homeomorphic. If the group is torsion free, it can be identified with the fundamental groups of both surfaces and hence (by Nielsen's theorem) the group determines a homotopy class of orientation-reversing homeomorphisms between the two surfaces.

In the case of the group $G^{\tau}$ defined by (8.1) the two surfaces are the mirror image $\bar{S}_{0}$ of $S_{0}=f_{0}^{-1}(\Sigma)$ and $S=f^{-1}(\Sigma)$, and the homotopy class is that of the mapping

$$
f^{-1} \circ f_{0} \circ j^{-1}
$$

where $j$ is the canonical mapping of $S_{0}$ onto $\bar{S}_{0}$ (and $\tau_{0}=\left[f_{0}: S_{0} \rightarrow \Sigma\right]$, $\tau=[f: S \rightarrow \Sigma])$. The verification is left to the reader.

(C) The simultaneous uniformization theorem [18], a generalization of the limit circle theorem by Klein-Poincaré-Koebe, asserts that given two Riemann surfaces of the same type $(p, n)$, and a homotopy class of orientation-reversing homeomorphisms between them, there exists a quasi-Fuchsian group representing these surfaces and this class. The group is determined uniquely, up to a conjugation in $\operatorname{PSL}(2, \mathrm{C})$.

The proof of the existence part of the theorem is essentially the construction of the group $G^{\tau}$ above. Briefly speaking, the desired group is obtained as a quasiconformal deformation of a Fuchsian group $G_{0}$, i.e., by conjugating $G_{0}$ by a $\mu$-conformal automorphism of $\mathbf{C}, \mu$ being chosen appropriately but so as to satisfy (6.5). The uniqueness proof is rather straightforward but relies on delicate properties of quasiconformal mappings. (For the proof, and a more general version of the theorem see [20].)

(D) Quasi-Fuchsian groups had been known to Klein and Poincaré. The classical construction starts with a sequence of circles $C_{1}, \ldots, C_{N}, C_{N+1}=$ $C_{1}$, such that $C_{j}$ touches or intersects $C_{j-1}$ and $C_{j+1}$ and no other circle, $j=1, \ldots, N$. The desired group is obtained as the subgroup of orientationpreserving transformations in the group generated by $N$ reflections about the circles $C_{1}, \ldots, C_{N}$. 
Also, Poincaré stated without proof that if one changes a little the generators of a Fuchsian group, preserving their types and all relations between them, but permitting the generators to become complex, one obtains a quasi-Fuchsian group.

The simultaneous uniformization theorem, however, was found only when the theory of quasiconformal mappings was sufficiently developed. As a matter of fact, no proof of the theorem not relying on quasiconformal mappings is known.

(E) The fixed curve of a quasi-Fuchsian group of the type considered here has many interesting properties. It admits a parametric representation by Hölder continuous functions and has measure zero. But it is not rectifiable, Bowen [37]. Indeed, Bowen proved that its Hausdorff dimension is strictly between 1 and 2 .

(F) An important theorem by Maskit [37] states that any finitely generated Kleinian group whose region of discontinuity has precisely two components is either quasi-Fuchsian and a quasiconformal deformation of a Fuchsian group, or $a \mathbf{Z}_{2}$ extension of a quasi-Fuchsian group.

For a simplified proof, see Kra and Maskit [76].

(G) The set of conjugacy classes of quasi-Fuchsian groups representing two Riemann surfaces of type $(p, n)$ is easily seen to be a $(6 p-6+2 n)$ dimensional complex manifold. By the results of $\$ 6$ it is isomorphic to $T_{p, n} \times T_{p, n}$.

\section{Fiber spaces over Teichmüller spaces.}

(A) We return to the embedding $T_{p, n} \subset B_{2}\left(L, G_{0}\right)$ of $\$ 6$ and note that to every $\tau \in T_{p, n}$ there belongs a Jordan domain

$$
D_{\tau}=w^{\mu}(U)
$$

bounded by the Jordan curve consisting of $z=\infty$ and the arc

$$
\zeta=w^{\mu}(x), \quad-\infty<x<+\infty .
$$

For every fixed $x \in \mathbf{R}, \zeta$ depends holomorphically on $\mu \in L_{\infty}(\mathbf{C})$; it is not changed if $\mu$ is replaced by, say, $\mu^{\prime}$ as long as $\mu^{\prime}$ defines the same $\tau$. We express this by saying that the Jordan domain $D_{\tau}$ depends holomorphically on $\tau$.

(B) The fiber space $F_{p, n}$ over $T_{p, n}$ is the set of pairs

$$
(\tau, z) \text { with } \tau \in T_{p, n}, z \in D_{\tau} \text {. }
$$

It is a $(3 p-2+n)$-dimensional domain of holomorphy and is canonically isomorphic to $T_{p, n+1}$, the projection $F_{p, n} \rightarrow T_{p, n}$ corresponding to "forgetting one puncture" [27].

(C) Set

$$
\begin{aligned}
& \delta(1)=p \quad \text { if } n=0, \quad \delta(1)=p+n-1 \quad \text { if } n>0, \\
& \delta(r)=(2 r-1)(p-1)+n(r-1) \quad \text { if } r>1 .
\end{aligned}
$$

For every $r$ there are $\delta(r)$ holomorphic functions $\Psi_{1}^{r}(\tau, z), \Psi_{2}^{r}(\tau, z), \ldots$ in $F_{p, n}$ such that for every fixed $\tau \in T_{p, n}$ the function $\Psi_{1}^{r}, \ldots, \Psi_{\delta(r)}^{r}$ form a basis 
of the space of cusp forms of weight $(-2 r)$ in $D_{\tau}$ for the group $G^{\tau}$. (A cusp form of weight $(-2 r)$ is a holomorphic function $\psi(z), z \in D_{\tau}$ such that $\psi(z) d z^{r}$ is $G^{\tau}$-invariant, i.e.

$$
\psi(g(z)) g^{\prime}(z)^{r}=\psi(z), \quad g \in G^{\tau},
$$

and

$$
\begin{gathered}
\iint_{D_{\tau} / G^{\tau}} \lambda_{\tau}(z)^{2-2 q}|\psi(z)| d x d y<\infty, \\
\sup \left|\lambda_{\tau}(z)^{-q} \psi(z)\right|<\infty,
\end{gathered}
$$

where $\lambda_{\tau}(z)|d z|$ is the Poincaré line element in $D_{\tau}$.)

(D) The existence of the functions $\Psi_{j}^{r}$ can be obtained from Grauert's theorem on holomorphic triviality of topologically trivial holomorphic vector bundles over Stein manifolds. For $r>1$ there is also an explicit integral formula which we will write down without discussing its derivation (which can be found in [21, 22, 24]).

If $\psi_{1}, \psi_{2}, \ldots, \psi_{\delta(r)}$ is a basis of cusp forms of weight $(-2 r)$ in $U$ for the group $G_{0}$, then (setting $\zeta=\xi+\sqrt{-1} \eta$ )

$$
\Psi_{j}^{r}(\tau, z)=\iint_{L} \frac{\eta^{2 r-2} \psi_{j}(\bar{\zeta})\left[\partial w^{\mu}(\zeta) / \partial \zeta\right]^{r}}{\left[w^{\mu}(\zeta)-z\right]^{2 r}} d \xi d \eta
$$

Here $w^{\mu}$ is the function defined in $\S 6$, (D); recall that $w^{\mu} \mid L$ is a holomorphic function depending only on $\tau$ and not on the particular choice of $\mu$.

(E) For $n=0$ and $r=3$ the functions $\Psi_{1}^{3}(\tau, z), \ldots, \Psi_{5 p-5}^{3}$ are holomorphic functions on $F_{p, 0}$ with the property: every algebraic curve of genus $p$ admits a parametric representation by rational functions of these functions, for some fixed $\tau$.

(F) The group $G_{0}$ operates on $F_{p, n}$ by the rule $g_{0}((\tau, z))=\left(\tau, g^{\mu}(z)\right)$ where $g^{\mu}=w^{\mu} \circ g \circ\left(w^{\mu}\right)^{-1}$. The quotient $F_{p, n} / G_{0}$ is a complex manifold and a fiber space over $T_{p, n}$, the fiber over a point $\tau=[f: S \rightarrow \Sigma]$ being isomorphic to the Riemann surface $S$. The manifold $V_{p, n}=F_{p, n} / G_{0}$ has been called the universal Teichmüller curve.

Except for some special and rather obvious cases the universal Teichmüller curve admits no holomorphic sections. This has been first shown by Hubbard [67], for $n=0$, and then by Earle and $\mathrm{Kra}[50,51]$ for the general case. The proofs use essentially Royden's theorem.

(G) One can define [27] an extended modular $\operatorname{group}_{\bmod }$,n holomorphic self-mappings of $F_{p, n}$ which acts properly discontinuously and contains $G_{0}$ as a normal subgroup with $\operatorname{Mod}_{p, n}=\bmod _{p, n} / G_{0}$. The quotient

$$
Y_{p, n}=F_{p, n} / \bmod _{p, n}
$$

is a normal complex space fibered over the moduli space $X_{p, n}$, the fiber over a point $x \in X_{p, n}$ being $S / \operatorname{Aut}(S)$ where $S$ is a Riemann surface (unmarked) of type $(p, n)$ represented by $x$ and $\operatorname{Aut}(S)$ the finite group of conformal 
self-mappings of $S$. (This statement must be slightly modified if $(p, n)=$ $(1,1)(1,2)$ or $(2,0)$.)

The isomorphism theorem in (B) implies that $X_{p, n+1}$ is a ramified $(n+1)$ sheeted covering space of $Y_{p, n}$.

(H) Earle [4] constructed a fiber space over $T_{p, 0}$ whose fibers are Jacobian varieties of genus $p$. We refer to his paper for details.

\section{Complex boundaries.}

(A) We proceed to discuss the complex boundary $\partial T_{p, n}$ defined by the embedding $T_{p, n} \subset B_{2}\left(L, G_{0}\right)$, see $\S 6$. This boundary depends, of course, on the choice of the origin $\tau_{0}$, cf. Bers [25], Maskit [84], Abikoff [2].

It is now convenient to associate with every cusp form $\varphi \in B_{2}(L, G)$ the function

$$
W_{\varphi}(z)=\eta_{1}(z) / \eta_{2}(z), \quad z \in L,
$$

where $\eta_{1}$ and $\eta_{2}$ satisfy

$$
2 \eta_{j}^{\prime \prime}(z)+\varphi(z) \eta_{j}(z)=0, \quad j=1,2, \quad z \in L,
$$

and

$$
\eta_{1}(-i)=\eta_{2}^{\prime}(-i)=1, \quad \eta_{1}^{\prime}(-i)=\eta_{2}(-i)=0 .
$$

This function, just as the function $w^{\mu} \mid L$ defined in $\S 6,(\mathrm{C})$ has the Schwarzian derivative $\varphi=\varphi^{\mu}$. But while $w^{\mu} \mid L$ is defined only for $\varphi \in T_{p, n} \subset B_{2}\left(L, G_{0}\right)$ and is normalized by the "boundary conditions" $w^{\mu}(0)=0, w^{\mu}(1)=1, w^{\mu}(\infty)$ $=\infty$, the function $W_{\varphi}$ is defined for all $\varphi \in B_{2}(L, G)$ and is normalized by the initial condition $W_{\varphi}(t-i)=t^{-1}+0(t), t \rightarrow 0$. Of course, for $\varphi \in T_{p, n}$, the function $W_{\varphi}$ is of the form $\alpha \circ w^{\mu} \mid L$ where $\alpha \in P S L(2, C)$, but $\alpha$ depends on $\varphi$ and need not have a limit as $\varphi$ converges to $\partial T_{p, n}$.

(B) For every $\varphi \in B_{2}\left(L, G_{0}\right)$ there is a homomorphism

$$
G_{0} \ni g \mapsto \chi_{\varphi}(g) \in \operatorname{PSL}(2, \mathrm{C})
$$

such that $W_{\varphi} \circ g=\chi_{\varphi}(g) \circ W_{\varphi}$. The group $\chi_{\varphi}\left(G_{0}\right)=G^{\varphi}$ is the so called monodromy group of the differential equation (10.2).

For $\varphi \in T_{p, n}$ the function $W_{\varphi}$ is univalent meromorphic; its image contains the point at infinity. The same is true for $\varphi \in \partial T_{p, n}$, but $W_{\varphi}(L)$ is a Jordan domain only if $\varphi \in T_{p, n}$.

For $\varphi \in T_{p, n} \cup \partial T_{p, n}$ the homomorphism $\chi_{\varphi}$ is an isomorphism and

$$
G^{\varphi}=W_{\varphi} G_{0} W_{\varphi}^{-1} \text {. }
$$

For $\varphi \in T_{p, n}$ the group $G^{\varphi}$ is quasi-Fuchsian, with precisely one unbounded component (a component of a Kleinian group is a component of its domain of discontinuity). The quotient of the unbounded component by $G^{\varphi}$ is always $\bar{S}_{0}$, that of the bounded component the "variable" Riemann surface $S=f_{\tau}^{-1}(\Sigma), \tau$ being the point in Teichmüller space represented by $\varphi$. (For the sake of brevity we often say "is" instead of "is conformally equivalent to".)

(C) It turns out that for $\varphi \in \partial T_{p, n}$, the group $G^{\varphi}$ is always Kleinian and has 
a single invariant component $\Delta_{0}=W_{\varphi}(L)$; this component contains the point at infinity and $\Delta_{0} / G^{\varphi}$ is $\bar{S}_{0}$.

Any other component $\Delta$ of $G^{\varphi}$ is simply connected, not invariant (i.e., there is a $g \in G^{\varphi}$ with $g(\Delta) \neq \Delta$ ), and the stabilizer $G_{\Delta}^{\varphi}$ of $\Delta$ in $G^{\varphi}$ always contains an accidental parabolic element, i.e., a parabolic element $\chi_{\varphi}(g)$ with $g \in G_{0}$ hyperbolic. Also, $\Delta / G_{\Delta}^{\varphi}$ is a Riemann surface of some type $(\hat{p}, \hat{n})$.

(D) It is not difficult to verify that points $\varphi \in B_{2}\left(L, G_{0}\right)$, with $\chi_{\varphi}(g)$ parabolic for some hyperbolic $g \in G_{0}$, lie on countably many holomorphic hypersurfaces in $B_{2}\left(L, G_{0}\right)$. The union of these hypersurfaces has real codimension 2, while $\partial T_{p, n}$ has real codimension 1 . Hence $W^{\varphi}(L)$ is the only component of $G^{\varphi}$ for all $\varphi \in \partial T_{p, n}$, except for a subset of positive real codimension.

(E) Now let $\varphi \in \partial T_{p, n}$ belong to this small set. Then there is a finite nonempty set of noninvariant components $\Delta_{1}, \ldots, \Delta_{N}$ of $G^{\varphi}$ such that $g\left(\Delta_{j}\right) \neq \Delta_{k}$ for $j \neq k$ and $g \in G^{\varphi}$, and every component of $G^{\varphi}$, distinct from the invariant component $\Delta_{0}$, is of the form $g\left(\Delta_{j}\right)$ for some $g \in G^{\varphi}$ and some $j$, $1 \leqslant j \leqslant N$. If $\Omega$ denotes the region of discontinuity of $G^{\varphi}$, then

$$
\Omega / G^{\varphi}=\Delta_{0} / G^{\varphi}+\Delta_{1} / G_{\Delta_{1}}^{\varphi}+\cdots+\Delta_{N} / G_{\Delta_{N}}^{\varphi}=\bar{S}_{0}+S_{1}+\cdots+S_{N} .
$$

It can be shown that there are on $S_{0}$ finitely many Jordan curves, which can be chosen as Poincaré geodesics $C_{1}, \ldots, C_{r}, 0<r \leqslant 3 p-3+n$, with the following property. The complement of $\left(C_{1} \cup \cdots \cup C_{n}\right)$ in $S_{0}$ has $M \geqslant N$ components $D_{1}, \ldots, D_{M}$ and these can be ordered so that $D_{j}$ is homeomorphic to $S_{j}, j=1, \ldots, N$.

(F) A boundary point $\varphi \in \partial T_{p, n}$ with $M=N>0$ is called regular and so is the group $G^{\varphi}$.

Such a group may be said to represent the mirror image $\bar{S}_{0}$ of the reference surface $S_{0}$ and one (if $N=1$ ) or several (if $N>1$ ) surfaces obtained by drawing $r>0$ disjoint geodesic Jordan arc on $S_{0}$ and then "squeezing" each of those into a point (which may be thought of as two punctures joined into a node).

All combinatorially possible regular $G^{\varphi}$ actually occur (Maskit, Abikoff). Furthermore, if $\varphi$ is a regular point on $\partial T_{p, n}$ and the surfaces $S_{1}, \ldots, S_{N}$ have types $\left(p_{1}, n_{1}\right), \ldots,\left(p_{N}, n_{N}\right)$, then there is a complex manifold $A \subset \partial T_{p, n}$, containing $\varphi$, of dimension

$$
3\left(p_{1}+\cdots+p_{N}\right)-3 N+n_{1}+\cdots+n_{N},
$$

which is isomorphic to the product $T_{p_{1} n_{1}} \times T_{p_{2} n_{2}} \times \cdots \times T_{p_{N} n_{N}}$.

(G) A boundary point $\varphi \in \partial T_{p, n}$ is called partially degenerate if $M>N>$ 0 , and so in the group $G^{\varphi}$. The group represents, besides $\bar{S}_{0}$, some but not all surfaces obtained by squeezing $r>0$ disjoint geodesic Jordan curves on $S_{0}$ into punctures. It is worth noting that no thrice punctured sphere may be "thrown away". 
Abikoff showed that all combinatorially possible types of partially degenerate groups $G^{\varphi}$ occur as boundary points of the boundary Teichmüller spaces $A$ described above.

(H) A point $\varphi \in \partial T_{p, n}$ and the group $G^{\varphi}$ are called totally degenerate if $G^{\varphi}$ has only the component $W_{\varphi}(L)$ and thus represent only $\bar{S}_{0}$. We already noted that a boundary group $G^{\varphi}$ without accidental parabolic elements must be totally degenerate. However, there are also totally degenerate groups with accidental parabolic elements.

\section{1. $b$-groups.}

(A) A finitely generated Kleinian group $G$ is called a b-group if it has precisely one invariant component $\Delta_{0}$, and $\Delta_{0}$ is simply connected. Groups $G^{\varphi}, \varphi \in T_{p, n}$, are $b$-groups and the name " $b$-group" was chosen in the hope that each such group appears on the boundary of some Teichmüller space. If $G$ contains elliptic elements, the Teichmüller space in question must be a Teichmüller space of a Fuchsian group with torsion (see §19). Torsion requires no essentially new ideas and we will consider here only torsion-free $b$-groups.

(B) By Ahlfors' finiteness theorem [11, 73] a $b$-group represents finitely many Riemann surfaces, each with a finite Poincare area. By the "second area inequality", see [73], the total Poincaré area of $\Omega / G$, where $\Omega$ is the region of discontinuity of $G$, is at most twice the Poincaré area of $\Delta_{0} / G$. (In $\$ 10$, (E) we encountered this inequality in the guise $M \geqslant N$.) If equality holds, $G$ is called regular, and Abikoff proved that a regular b-group is always a boundary group.

(C) The existence of finitely generated Kleinian groups with a connected and simply connected region of discontinuity (totally degenerate groups) seems not to have been known to the founding fathers of the theory, Klein and Poincaré. The existence proof for such groups sketched in $\$ 10$, (D) actually yields the following results: given a Riemann surface $S$ of type $(p, n)$, with $3 p-3+n>0$, there exist continuously many nonconjugate torsion-free totally degenerate Kleinian groups $G$, with region of discontinuity $\Omega$ and $\Omega / G$ conformally equivalent to $S$.

(D) A remarkable property of degenerate groups has been discovered by L. Greenberg. It refers to the interpretation of Kleinian groups as discrete groups of non-Euclidean motions in space. This interpretation, due to Poincaré plays an increasingly important part in recent work on Kleinian groups. In the hands of Thurston it became an amazingly powerful tool for investigating discrete groups and hyperbolic structures on 3-manifolds.

Call a quaternion $x+y i+t j+s k$ special if $t>0, s=0$, and write a special quaternion as $Z=z+t j, z=x+i y \in \mathbf{C}$. The Poincaré half-space $\mathbf{H}_{3}$ is the set of special quaternions (see $\S 1,(C)$ ); with the metric (1.2) it becomes a model of non-Euclidean space. Every Möbius transformation

$$
z \mapsto g(z)=(a z+b) /(c z+d),
$$


where $a, b, c, d \in \mathbf{C}$ and $a d-b c=1$, gives rise to an isometry of $\mathbf{H}_{3}$ which we denote by the same letter

$$
Z \mapsto g(Z)=(a Z+b)(c Z+d)^{-1},
$$

and every orientation-preserving isometry of $\mathbf{H}_{3}$ can be so written. (Similarly, every conformal self-mapping of $\mathbf{H}_{n}$ can be interpreted as an orientation-preserving isometry of $\mathbf{H}_{n+1}$, but the elegant formula involving quaternions is restricted to $n=2$.)

(E) It is classical that every discrete finitely generated group of nonEuclidean motions in the plane, i.e., every finitely generated Fuchsian group, has a fundamental domain which is a non-Euclidean polygon with finitely many sides. Greenberg [61] showed that this is not so in space. $A$ degenerate Kleinian group, viewed as a group of motions in $\mathbf{H}_{3}$, does not have a finitelymany-sided fundamental polyhedron. It is as one says, geometrically infinite. So also is every partially degenerate boundary group.

(F) The difference between geometrically finite and geometrically infinite Kleinian groups dominates the whole theory. In general, geometrically finite groups are easier to investigate. Maskit $[87,88]$ gave a complete classification of all such groups with an invariant component.

All finitely generated quasi-Fuchsian groups and regular boundary groups are geometrically finite.

\section{Ahlfors' problem, Sullivan's theorem and the complex boundary.}

(A) In a seminal paper [11] on Kleinian groups Ahlfors asked whether the limit set $\Lambda(G)$ of a finitely generated Kleinian group has area (2-dimensional Lebesgue measure) zero. Later [12] he proved that this is so if the group is geometrically finite. (The condition of being finitely generated is essential; Abikoff [1] constructed an infinitely generated quasi-Fuchsian group whose fixed curve has positive area.) Thurston showed that limit sets of so-called strong limits of (finitely generated) quasi-Fuchsian groups also have vanishing area (cf. [118] $\$ 8.12 .4$ and $\$ 9.2$ ). This includes all groups on $\partial T_{p, n}$ without accidental parabolic elements.

(B) The importance of Ahlfors' problem for function theory derives from the following question. Suppose $G$ is a finitely generated Kleinian group, $w$ a quasiconformal self-mapping of $\mathbf{C} \cup\{\infty\}$ which is compatible with $G$, that is such that $w G w^{-1}$ is again a Kleinian group, and $\mu=(\partial w / \partial \bar{z}) /(\partial w / \partial z)$ the Beltrami coefficient of $w$. Is $w$ determined, except for a conjugation, by knowing $\mu \mid \Omega$, that is by knowing how $w$ changes the conformal structure of the Riemann surfaces represented by $G$ ?

The answer is yes if mes $\Lambda(G)=0$. In an important recent paper [113] Sullivan showed that the answer is always yes.

(C) We ask now (as we asked for the real boundaries in §4) whether the modular group $\operatorname{Mod}_{p, n}$ acts (by continuity) on $\partial T_{p, n} \subset B_{2}\left(L, G_{0}\right)$. A partial answer [32] can be given using Sullivan's theorem. 
A boundary group $G^{\varphi}, \varphi \in \partial T_{p, n}$, represents the fixed Riemann surface $\bar{S}_{0}$ and $N \geqslant 0$ other Riemann surfaces of types $\left(p_{1}, n_{1}\right), \ldots,\left(p_{N}, n_{N}\right)$. We say that $\varphi$ has no moduli if either $N=0$ or $\left(p_{j}, n_{j}\right)=(0,3)$ for $j=1, \ldots, N$.

The modular group acts (by continuity) at all boundary points with no moduli.

This includes all degenerate, some regular and some partially degenerate boundary points. (It includes the whole boundary if $p=n=1$.)

(D) If $\varphi \in \partial T_{p, n}$ has moduli, $\left\{\varphi_{\nu}\right\} \subset T_{p, n}$ and $\varphi_{\nu} \rightarrow \varphi$, and if $\gamma \in \operatorname{Mod}_{p, n}$ and a subsequence $\left\{\varphi_{\nu_{i}}\right\}$ are such that $\gamma\left(\varphi_{\nu_{i}}\right) \rightarrow \varphi_{1} \in \partial T_{p, n}$, then $G^{\varphi_{1}}$ represents as many surfaces as $G^{\varphi}$ does, and of the same types.

(E) If $\gamma \in \operatorname{Mod}_{p, n}$ leaves the origin $\tau_{0}=\left[f_{0}: S_{0} \rightarrow \Sigma\right]$ fixed, that is, if $\gamma=\omega_{*}$ where $\omega: \Sigma \rightarrow \Sigma$ can be written as $f_{0} \circ h \circ f_{0}^{-1}$ with $h: S_{0} \rightarrow S_{0}$ conformal, then $\gamma$ acts continuously on all of $\partial T_{p, n}$. Indeed, in this case $\gamma$ is the restriction to $T_{p, n}$ of a linear transformation on $B_{2}\left(L, G_{0}\right)$.

(F) According to a forthcoming paper by Kerckhoff and Thurston some $\gamma \in \operatorname{Mod}_{p, n}$ must fail to act on some regular points on $\partial T_{p, n}$ (provided that $\left.\operatorname{dim} T_{p, n}>1\right)$.

\section{The Maskit embedding.}

(A) Besides the injection $T_{p, n} \hookrightarrow \mathbf{C}^{3 p-3+n}$ described in $\$ 6$ there is another injection due to Maskit [86] which depends not on the choice of an origin in $T_{p, n}$, which involves continuously varying parameters, but on finitely many choices. We describe it for the case $T_{p}=T_{p, 0}$, the case considered by Maskit. The extension to $n \neq 0$ involves neither new ideas nor technical difficulties.

In order to define the Maskit embedding one chooses $3 p-3$ disjoint and homotopically independent Jordan curves $C_{1}, \ldots, C_{3 p-3}$ on $\Sigma$ (which is now a compact surface of genus $p$ ); this can be done in only finitely many topologically distinct ways.

The idea of the Maskit embedding is to represent a point $\tau=[f: S \rightarrow \Sigma]$ of $T_{p}$ by a regular $b$-group $G$ with invariant component $\Delta_{0}$, which represents $S=\Delta_{0} / G$, and $2 p-2$ thrice punctured spheres. This group is to have the property that the lift of each curve $f^{-1}\left(C_{j}\right), j=1, \ldots, 3 p-3$, to $\Delta_{0}$ is invariant under an accidental parabolic element of $G$.

(B) The Maskit coordinates are $(3 p-3)$-tuples of numbers $Z_{j} \in U$, belonging to a domain $\mathfrak{D} \subset U^{3 p-3}$. The numbers $Z_{1}, \ldots, Z_{3 p-3}$ determine a sequence of generators for the group $G$. The technical details are somewhat complicated and will be explained later (cf. $\$ 16$, especially (F)), in a more general context.

(C) The boundary of $T_{p}$ in the Maskit embedding, and the action of $\operatorname{Mod}_{p}=\operatorname{Mod}_{p, 0}$ on this boundary, have not yet been investigated sufficiently. We mention, however, that if $\omega$ is a Dehn twist about $C_{j}$, $\omega_{*}$ leaves all $Z_{k}$, $k \neq j$, alone and replaces $Z_{j}$ by $Z_{j}+1$.

\section{Riemann surfaces with nodes.}

(A) We shall now describe an extension of Teichmüller space theory in which compact Riemann surfaces are replaced by Riemann surfaces with 
nodes. The theory had been announced almost ten years ago [28, 29] but the promised detailed presentation has . ot yet appeared. The author uses this occasion to present his apologies and to express the hope that the long delayed obligation will be fulfilled in the not too distant future.

(B) A compact surface with nodes $\Sigma$ is a compact Hausdorff space such that every point $P \in \Sigma$ has a fundamental system of neighborhoods homeomorphic either to the disc $|z|<1$ in $\mathbf{C}$ or to the set $\left|z_{14}<1,\right| z_{2} \mid<1, z_{1} z_{2}=0$ in $\mathbf{C}^{2}$. In the second case $P$ is called a node.

Each component of the complement of the set of nodes is called a part of $\Sigma$, and $\Sigma$ is called oriented if every part is, stable if no part has an Abelian fundamental group, nonsingular if there are no nodes.

We shall consider only stable oriented $\Sigma$.

The genus $p$ of $\Sigma$ is the genus of the nonsingular surface obtained by thickening each node; the stability condition implies that $p>1$.

(C) A (compact stable) Riemann surface with nodes $S$ is a (compact stable) surface with nodes such that every part $S_{j}$ of $S$ is a Riemann surface of some type $\left(p_{j}, n_{j}\right)$. The stability condition implies that $2 p_{j}-2+n_{j}>0$ so that each part of $S$ has a Poincaré metric. The total Poincaré area of $S$ is $2 \pi(2 p-2)$ as for a nonsingular surface.

(D) A regular $r$-differential on $S$ is a rule which assigns a holomorphic $r$-differential (holomorphic section of $r$ times the canonical line bundle) to every part $S_{j}$ of $S$, with the provision that at the punctures the $r$-differential have poles at most of order $r$, and at any two punctures joined in a node the residues are equal, if $r$ is even, or opposite, if $r$ is odd.

(DEFINITION OF RESIDUE. Let $q$ be an $r$-differential which has at $P$ a pole of order at most $r$. Either the order is less than $r$ and the residue at $P$ is 0 , or there is a local parameter $\zeta$ with $\zeta=0$ at $P$ and $q=a(d \zeta / \zeta)^{r}$ near $P$ and the residue at $P$ is $a$.)

The dimension $\delta(r)$ of the space of regular $r$-differentials on $S$ is

$$
\delta(r)= \begin{cases}p & \text { if } r=1 \\ (2 r-1)(p-1) & \text { if } r>0\end{cases}
$$

as for nonsingular surfaces. For $r>2$ one can use a basis of regular $r$-differentials to embed $S$ into $\mathbf{P}_{\delta(r)-1}$ and thus realize it as an algebraic curve of arithmetic genus $p$, with no singularities except for simple nodes.

(E) A strong deformation of a surface with nodes $\Sigma_{1}$ onto a surface with nodes $\Sigma_{2}$, both of the same genus $p$, is a continuous surjection $\Sigma_{1} \rightarrow \Sigma_{2}$ such that the image of a node of $\Sigma_{1}$ is a node $\Sigma_{2}$, the inverse image of a node of $\Sigma_{2}$ is a node of $\Sigma_{1}$ or a Jordan curve on a part of $\Sigma_{1}$, and the mapping restricted to the complement of the inverse images of all nodes is an orientation-preserving homeomorphism.

(F) We say that $\Sigma_{1}$ is terminal if it has the largest possible number of nodes, namely $3 p-3$. There are only finitely many topologically distinct terminal 
surfaces of a given genus $p$, and every strong deformation of a terminal surface is a homeomorphism.

(G) From now on the letter $\Sigma$ (or $S$ ), with or without subscripts and superscripts will denote a compact surface (Riemann surface) with nodes, of genus $p$.

\section{Strong deformation spaces.}

(A) Two strong deformations $f: S \rightarrow \Sigma$ and $f^{\prime}: S^{\prime} \rightarrow \Sigma$ will be called equivalent if there is a commutative diagram

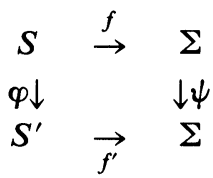

where $\varphi$ and $\psi$ are bijective homeomorphisms homotopic to an isomorphism and to the identity, respectively (cf. the definition in $\$ 2,(\mathrm{~A})$ ).

If $f: S \rightarrow \Sigma$ and $g: \Sigma \rightarrow \Sigma_{0}$ are strong deformations, so is $g \circ f$ and the equivalence class of $g \circ f$ depends only on the equivalence class of $f$ and the homotopy class of $g$.

(B) The strong deformation space $\mathfrak{D}(\Sigma)$ of $\Sigma$ is the set of all equivalence classes $[f: S \rightarrow \Sigma]$ of strong deformations $f: S \rightarrow \Sigma$.

Every strong deformation $g: \Sigma \rightarrow \Sigma_{0}$ induces a mapping $g_{*}: \mathfrak{D}(\Sigma) \rightarrow \mathfrak{D}\left(\Sigma_{0}\right)$ which takes $\left[f: S \rightarrow \Sigma\right.$ ] into [ $g \circ f: S \rightarrow \Sigma_{0}$ ]. The mappings $\omega_{*}$ induced by homeomorphisms $h: \Sigma \rightarrow \Sigma$ form the modular $\operatorname{group} \operatorname{Mod}(\Sigma)$.

From now on we shall omit the adjective "strong" when there is no danger of misunderstanding.

(C) In order to topologize $\mathfrak{D}(\Sigma)$, let us set, for every closed curve $C$ on a part of $S$,

$$
l_{S}(C)=\inf \left(\text { Poincaré length of } C^{\prime}\right)
$$

where $C^{\prime}$ runs over the free homotopy class of $C$. If $l_{S}(C)>0$, then $l_{S}(C)$ equals the length of the unique Poincaré geodesic freely homotopic to $C$. We also set $l_{S}(P)=0$ if $P$ is a point on $S$.

A set $A \subset \mathfrak{D}(\Sigma)$ is called open if for every point $[f: S \rightarrow \Sigma]$ in $A$ there are finitely many closed curves $C_{1}, \ldots, C_{r}$ on parts of $S$, and an $\varepsilon>0$, such that whenever the deformation $h: S_{1} \rightarrow S$ satisfies the conditions

$$
\begin{aligned}
& \left|l_{S_{1}}\left(h^{-1}\left(C_{j}\right)\right)-l_{S}\left(C_{j}\right)\right|<\varepsilon \text { for } j=1, \ldots, r, \\
& \left|l_{S_{1}}\left(h^{-1}(Q)\right)\right|<\varepsilon \text { for all nodes } Q \text { of } S,
\end{aligned}
$$

the point [ $f \circ h: S_{1} \rightarrow \Sigma$ ] of $\mathfrak{D}(\Sigma)$ belongs to $A$.

This definition implies that induced maps are continuous and open.

It also implies that if $\Sigma$ is nonsingular, $\mathfrak{D}(\Sigma)$ may be identified with $T_{p}$.

(D) Let $\Sigma_{0}$ be terminal, let $P_{1}, \ldots, P_{3 p-3}$ be the nodes of $\Sigma_{0}$ and $\sigma_{1}, \ldots, \sigma_{2 p-2}$ the parts of $\Sigma_{0}$, each of which is homeomorphic to a thrice punctured sphere. 
Each node $P_{j}$ has two banks (we forego a formal definition of this term) and each part $\sigma_{i}$ has three boundary banks. For each $i$ we order these three banks cyclically.

Let $\left[f: S \rightarrow \Sigma_{0}\right.$ ] be a point of $\mathfrak{D}\left(\Sigma_{0}\right)$. Without loss of generality we assume that $C_{j}=f^{-1}\left(P_{j}\right)$ is either a node of $S$ or a Poincaré geodesic and we set

$$
2 \pi r_{j}=\text { Poincaré length of } C_{j} \text {. }
$$

If $r_{j}>0$ there is on each bank of $C_{j}$ a distinguished point determined as follows. Assume the bank in question is $C_{j}^{+}=f^{-1}\left(b^{\prime}\right)$ where $b^{\prime}$ is a bank of a node of $\Sigma_{0}$, and that $C_{j}^{+}$and two inverse images $f^{-1}\left(b^{\prime \prime}\right)$ and $f^{-1}\left(b^{\prime \prime \prime}\right)$ of banks of nodes "bound" the inverse image $f^{-1}\left(\sigma_{i}\right)$ of a part of $\Sigma_{0}$. Assume also that $b^{\prime \prime \prime}$ precedes $b^{\prime}$ in the cyclic ordering chosen above. There is a unique Poincaré geodesic $K$ in $f^{-1}\left(\sigma_{i}\right)$ which joins $C_{j}^{+}$to $f^{-1}\left(b^{\prime \prime \prime}\right)$, is orthogonal to $C_{j}$, and is orthogonal to $C_{k}$ if $f^{-1}\left(b^{\prime \prime \prime}\right)$ is a bank of $C_{k}$ (rather than a node of $S$ ). The endpoint of $K$ on $C_{j}$ is the distinguished point on $C_{j}^{+}$.

If $r_{j}>0$, let $\theta_{j}$ be the smallest nonnegative number with the property: if we approach the distinguished point on one bank of $C_{j}$ along a geodesic arc in this bank turn left and proceed along $C_{j}$, we reach the second distinguished point after having covered the distance $\theta r_{j}$. Clearly $0 \leqslant \theta<2 \pi$.

Now set $z_{j}=0$ if $r_{j}=0$ and

$$
z_{j}=r_{j} e^{i \theta} \quad \text { if } r_{j}>0 .
$$

The numbers $z_{1}, \ldots, z_{3 p-3}$ depend only on the equivalence class $\left[f: S \rightarrow \Sigma_{0}\right]$; we call them the Fenchel-Nielsen coordintes of this point in $\mathscr{D}\left(\Sigma_{0}\right)$. It can be shown that the map

$$
\left[f: S \rightarrow \Sigma_{0}\right] \mapsto\left\{z_{1}, \ldots, z_{3 p-3}\right\}
$$

is a homeomorphism of $\mathfrak{D}\left(\Sigma_{0}\right)$ onto $\mathbf{C}^{3 p-3}$.

Note that the inverse image of a coordinate plane $z_{k}=0$ consists of all [ $f: S \rightarrow \Sigma_{0}$ ] such that $f^{-1}\left(P_{j}\right)$ is a node.

(E) It can be shown that for every $g$ : $\Sigma \rightarrow \Sigma_{0}, \Sigma_{0}$ as before, the induced map $g_{*}: \mathfrak{D}(\Sigma) \rightarrow \mathfrak{D}\left(\Sigma_{0}\right)$ is a universal covering of its image, the image being the set of those [ $f: S \rightarrow \Sigma_{0}$ ] for which $f^{-1}\left(P_{j}\right)$ is not a node whenever $g^{-1}\left(P_{j}\right)$ is not a node.

Hence $\mathfrak{D}(\Sigma)$ is homeomorphic to $\mathbf{C}^{3 p-3}$ and one can define Fenchel-Nielsen coordinates $w_{1}, \ldots, w_{3 p-3}$ in $\mathfrak{D}(\Sigma)$, defined by the deformation $g$, as complex numbers $w_{1}, \ldots, w_{3 p-3}$ such that

$$
\begin{aligned}
w_{j}=z_{j} & \text { if } g^{-1}\left(P_{j}\right) \text { is a node of } \Sigma, \\
e^{w_{j}}=z_{j} & \text { if } g^{-1}\left(P_{j}\right) \text { is not a node of } \Sigma .
\end{aligned}
$$

If $\Sigma$ is nonsingular, one obtains the coordinates in $T_{p}$ used by Fenchel and Nielsen in the famous manuscript.

(F) One now verifies easily that every induced map of one deformation space into another is a universal covering of the image.

(G) It is important to note that the Fenchel-Nielsen coordinates are written 
as complex numbers merely for convenience. They are real coordinates and do not reflect the natural complex structure of the deformation spaces, which we proceed to define.

\section{Complex structure of deformation spaces.}

(A) Let $\Sigma_{1}$ be a nonsingular surface of genus $p$ and $\Sigma$ any other surface. Since $\mathfrak{D}\left(\Sigma_{1}\right)$ is identical with $T_{p}$, see $\S 15$, (C), it is a complex manifold. Since there always is an induced map $g_{*}: \mathfrak{D}\left(\Sigma_{1}\right) \rightarrow \mathfrak{D}(\Sigma)$ which is a universal covering of the image $g_{*}\left(\mathfrak{D}\left(\Sigma_{1}\right)\right)$, see $\$ 15,(\mathrm{~F})$, the set $g_{*}\left(\mathfrak{D}\left(\Sigma_{1}\right)\right) \subset \mathfrak{D}(\Sigma)$ has a natural structure of a complex manifold, and since $\mathfrak{D}(\Sigma)-g_{*}\left(\mathfrak{D}\left(\Sigma_{1}\right)\right)$ is nowhere dense (cf. $\$ 15,(\mathrm{D})$ ), $\mathfrak{D}(\Sigma)$ has a ringed structure: a continuous function on an open set $A \subset \mathfrak{D}(\Sigma)$ is called holomorphic if its restriction to $A \cap g_{*}\left(\mathscr{D}\left(\Sigma_{1}\right)\right)$ is.

Note that induced maps are holomorphic with respect to this ringed structure.

Actually $\mathfrak{D}(\Sigma)$ is a complex manifold which can be realized as a bounded domain in $\mathbf{C}^{3 p-3}$.

We sketch the (lengthy) proof of this theorem below.

(B) First we consider a terminal surface $\Sigma_{0}$ with nodes $P_{1}, \ldots, P_{3 p-3}$ and parts $\sigma_{1}, \ldots, \sigma_{2 p-2}$.

We draw $2 p-2$ circular discs $\Delta_{1}, \ldots, \Delta_{2 p-2}$ in $\mathbf{C}$ and choose on each boundary $\partial \Delta_{j}$ points $Q_{j^{1}}, Q_{j^{2}}, Q_{j^{3}}$ which follow each other in this order, in the counterclockwise direction. We denote by $\gamma_{j^{r}}, j=1, \ldots, 2 p-2, \nu=1,2,3$, parabolic Möbius transformations with fixed points at $Q_{j^{\nu}}$ such that

$$
\gamma_{j^{3}} \circ \gamma_{j^{2}} \circ \gamma_{j^{1}}=\mathrm{id} \text {. }
$$

Then $\gamma_{j} 1, \gamma_{j^{2}}, \gamma_{j^{3}}$ generate a torsion-free Fuchsian group $\Gamma_{j}$ with limit set $\partial \Delta_{j}$. Both $\Delta_{j} / \Gamma_{j}$ and $\bar{\Delta}_{j} / \Gamma_{j}$, where $\bar{\Delta}_{j}$ is the domain exterior to $\partial \Delta_{j}$, are triply punctured spheres.

We assume that the $\Delta_{j}$ are so far apart that there are fundamental domains $F_{j}$ for $\Gamma_{j}$ in $\bar{\Delta}_{j}$ such that the closure of the complement of $F_{j}$ lies in the intersection of all $F_{k}, j \neq k$. Then, according to Klein's combination theorem, the group $\Gamma$ generated by $\Gamma_{1}, \ldots, \Gamma_{2 p-2}$ is their free product and Kleinian. It has precisely one invariant component $A_{0}$, not simply connected, while every noninvariant component is of the form $\gamma\left(\Delta_{j}\right)$ for some $j=1, \ldots, 2 p-2$ and $\gamma \in \Gamma$, and has $\gamma^{-1} \Gamma_{j} \gamma$ as stabilizer. Also, $A_{0} / \Gamma$ is a sphere with $6 p-6$ punctures.

For later use we denote by $\alpha_{j}$ the Möbius transformation which maps $\Delta_{j}$ onto $U$ taking $Q_{j^{\nu}}$ into $\infty$ and the other two points $Q$ on $\partial \Delta_{j}$ into 0 and 1 . Then $\alpha_{j^{\nu}} \Gamma_{j} \alpha_{j^{\nu}}^{-1}$ is the principal congruence subgroup modulo 2 of $\operatorname{PSL}(2, \mathrm{Z})$, and

$$
\alpha_{j^{\nu}} \circ \gamma_{j^{\nu}} \circ \alpha_{j^{\nu}}^{-1}(z)=z-2 .
$$

Next we divide the $6 p-6$ points $Q_{j}$, into $3 p-3$ disjoint ordered pairs, each pair being assigned to a node $P_{i}$ of $\Sigma_{0}$, in such a way that to a $P_{i}$ which lies in the closures of $\sigma_{j}$ and $\sigma_{j^{\prime}}, j<j^{\prime}$, belongs a pair $\left(Q_{j^{\prime}}, Q_{j^{\prime \prime}}\right)$, and to a $P_{i}$ which lies in the closure of one part $\sigma_{j}$ only belongs a pair $\left(Q_{j^{\nu}}, Q_{j^{v}}\right), \nu<\nu^{\prime}$. 
Note that if, for every ordered pair $\left(Q_{j^{*}}, Q_{j^{\prime \prime}}\right)$, we join the punctures of the Riemann surfaces $\Delta_{j^{\nu}} / \Gamma_{j}$ and $\Delta_{j^{\prime \nu^{\prime}}} / \Gamma_{j}$ corresponding to $Q_{j^{\prime}}$ and $Q_{j^{\prime \prime}}$, respectively, into a node, we obtain a Riemann surface with nodes $S_{0}$ homeomorphic to $\Sigma_{0}$.

We shall now thicken some of the $3 p-3$ nodes of $S_{0}$, in a way which depends on $3 p-3$ complex parameters.

(C) Let $\zeta_{1}, \ldots, \zeta_{3 p-3}$ be complex numbers with $\left|\zeta_{i}\right|<1$, and set

$$
\begin{aligned}
& \delta_{i}(z)=z \quad \text { if } \zeta_{i}=0, \\
& \delta_{i}=\alpha_{j^{\nu}}^{-1} \circ \beta_{i} \circ \alpha_{j^{\prime \prime}} \text { if } \zeta_{i} \neq 0
\end{aligned}
$$

where $\left(Q_{j^{\nu}}, Q_{j^{\prime \prime}}\right)$ belongs to $P_{i}$ and

$$
\beta_{i}(z)=-z-\sqrt{-1} \log \zeta_{i}
$$

where we use some determination of the logarithm. Note that for $\zeta_{i} \neq 0$ the Möbius transformation $\delta_{i}$ conjugates the cyclic group generated by $\gamma_{j^{\prime \prime}}$ into that generated by $\gamma_{j^{\prime}}$.

We say that $\left(\zeta_{1}, \ldots, \zeta_{3 p-3}\right)$ belongs to $\mathfrak{D}^{\prime}\left(\Sigma_{0}\right)$ if for every $P_{i}$ with $\zeta_{i} \neq 0$ to which there is associated the pair $\left(Q_{j^{\nu}}, Q_{j^{\prime \nu}}\right)$ there are Jordan curves $K_{j^{\prime}}, K_{j^{\prime \prime}}$ with the following properties: (a) $K_{j^{\nu}}$ contains the point $Q_{j^{\prime}}$ (and $K_{j^{\prime \prime}}$ contains the point $Q_{j^{\prime}}$ ), lies otherwise in $\Delta_{j}$ (in $\Delta_{j^{\prime}}$ ) and is invariant under $\gamma_{j^{\prime}}$ (under $\left.\gamma_{j^{\prime \prime}}\right)$; (b) the transformation $\delta_{i}$ maps the domain exterior to $K_{j^{\prime \prime}}$ onto the domain interior to $K_{j}$, and (c) the curves $K$ lying in a fixed $\Delta_{j}$ are taken, by the canonical projection $\Delta_{j} \rightarrow \Delta_{j} / \Gamma_{j}$, into disjoint Jordan curves.

One can verify that the condition

$$
\left(\zeta_{1}, \ldots, \zeta_{3 p-3}\right) \in \mathfrak{D}^{\prime}\left(\Sigma_{0}\right)
$$

does not depend on the choice of the discs $\Delta_{j}$ or the points $Q_{j}$. Nor does it depend on the determination of the logarithms in (16.1). Finally, (16.2) always holds if all $\left|\zeta_{i}\right|$ are sufficiently small for then the curves $K$ can be chosen as horocycles.

The group $\mathbb{S}=\mathbb{S}\left(\zeta_{1}, \ldots, \zeta_{3 p-3}\right)$ generated by $\gamma$ and $\delta_{1}, \ldots, \delta_{3 p-3}$ also does not depend on the determination of the logarithms.

(D) Under condition (16.2) the group \&S is Kleinian, in view of Maskit's second combination theorem, cf. [83, 85].

Assume that this is so and let $N$ denote the number of components of the space obtained from $\Sigma_{0}$ by removing all nodes $P_{i}$ with $\zeta_{i}=0$.

One can show that \&S has precisely $N+1$ nonconjugate components $A_{0}$, $A_{1}, \ldots, A_{n}$, none of which is invariant; the component $A_{0}$ is the domain bounded by the $2 p-2$ circles $\partial \Delta_{j}$. Let $\mathscr{S}_{t}$ denote the stabilizer of $A_{t}$ in $\mathbb{S}$. Then $\mathscr{G}_{0}=\Gamma$ and for $t>0$ each $\mathscr{S}_{t}$ is a regular $b$-group and if $\left(p_{t}, n_{t}\right)$ is the type of $A_{t} / \mathscr{G}_{t}$, then $n_{1}+\cdots+n_{N}=2 k$, where $k$ is the number of zeros among $\zeta_{1}, \ldots, \zeta_{3 p-3}$.

If we join any two punctures on the surfaces $A_{1} / \mathscr{S}_{1}, \ldots, A_{N} / \mathscr{S}_{N}$, which correspond to points $Q_{j^{\prime}}$ and $Q_{j^{\prime \prime}}$ belonging to a $P_{i}$ with $\zeta_{i}=0$, into a node, we obtain a compact Riemann surface $S$ of genus $p$ with $k$ nodes. 
The curves $K$ project into $3 p-3-k$ disjoint Jordan curves on $S$. Together with the nodes they divide $S$ into $2 p-2$ triply connected domains $s_{1}, \ldots, s_{2 p-2}$ which are in a natural bijection with the parts $\sigma_{1}, \ldots, \sigma_{2 p-2}$ of $\Sigma_{0}$. There is a deformation $f: S \rightarrow \Sigma_{0}$ such that $f\left(s_{i}\right)=\sigma_{i}$ for all $i$, and the point $\left[f: S \rightarrow \Sigma_{0}\right]$ in $\mathfrak{D}(\Sigma)$ depends only on the numbers $\zeta_{1}, \ldots, \zeta_{3 p-3}$ (and not on the choice of the curves $K$ ).

We have thus defined a mapping $\mathfrak{D}^{\prime}\left(\Sigma_{0}\right) \rightarrow \mathfrak{D}\left(\Sigma_{0}\right)$. It turns out that this is a bijective holomorphic (with respect to the ringed structure of $\mathfrak{D}\left(\Sigma_{0}\right)$ ) homeomorphism.

(E) We know now that $\mathscr{D}\left(\Sigma_{0}\right)$, for a terminal $\Sigma_{0}$, is a bounded domain in $\mathbf{C}^{3 p-3}$. For every node $P_{i}$ of $\Sigma_{0}$ the set $R_{i}$ of points [ $\left.f: S \rightarrow \Sigma_{0}\right]$ in $\mathfrak{D}\left(\Sigma_{0}\right)$ such that $f^{-1}\left(P_{i}\right)$ is a node is the intersection of $\mathfrak{D}\left(\Sigma_{0}\right)$ with the hyperplane $\zeta_{j}=0$.

For every $\Sigma$ there is a terminal $\Sigma_{0}$ and a subset $I$ of $\{1, \ldots, 3 p-3\}$ such that there is a deformation $g: \Sigma \rightarrow \Sigma_{0}$ which induces a universal covering

$$
g_{*}: \mathfrak{D}(\Sigma) \rightarrow \mathfrak{D}\left(\Sigma_{0}\right)-\bigcup_{i \in I} R_{i} .
$$

We conclude that $\mathfrak{D}(\Sigma)$ is a complex manifold and that there are complex coordinates $Z_{1}, \ldots, Z_{3 p-3}$ in $\mathfrak{D}(\Sigma)$ such that

$$
\begin{gathered}
Z_{i}=\zeta_{i} \quad \text { if } i \in I, \\
e^{2 \pi \sqrt{-1}} Z_{i}=\zeta_{i} \quad \text { if } i \notin I .
\end{gathered}
$$

Thus $\mathfrak{D}(\Sigma)$ is a domain in the product of $|I|$ unit discs and $3 p-3-|I|$ half-planes. $(|I|$ is the cardinality of $I$.)

In particular, if $\Sigma$ is nonsingular, $\mathscr{D}(\Sigma)=T_{p},|I|=0$ and $Z_{1}, \ldots, Z_{3 p-3}$ are the Maskit coordinates (cf. $\$ 13,(\mathrm{~B})$ ).

(F) It can be shown that for every $\Sigma, \mathfrak{D}(\Sigma)$ is a domain of holomorphy. Using this one can prove that for every $r=1,2, \ldots$, there is a trivial complex vector bundle over $\mathfrak{D}(\Sigma)$ such that the fiber over a point $[f: S \rightarrow \Sigma]$ is the space of regular $r$-differentials on $S$.

\section{Moduli spaces.}

(A) Let $\hat{X}_{p}$ denote the space of moduli (isomorphism classes) of compact Riemann surfaces with nodes, of genus $p$. This space can be topologized the same way as $\mathfrak{D}(\Sigma)$ was topologized in $\S 15$, (C). It follows easily that $X_{p}=X_{p, 0}$ the space of moduli of nonsingular Riemann surfaces of genus $p$, is an open dense subset of $\hat{X}_{p}$. Since $X_{p}$ is a normal complex space $(\mathrm{cf} . \S 6,(\mathrm{H})), \hat{X}_{p}$ has a natural ringed structure.

Studying the natural maps of all deformation spaces of genus $p$ into $\hat{X}_{p}$ one can show that $\hat{X}_{p}$ is a normal complex space (and a $V$-manifold).

(B) We now state a useful à priori inequality.

Let $S$ be a compact Riemann surface of genus $p$ with $k \geqslant 0$ nodes. One can draw $3 p-3-k$ disjoint (Poincaré) geodesic Jordan curves on the parts of $S$, none of which has length exceeding a constant $\alpha_{p}$ depending only on $p$.

This was first conjectured by Mumford. The author's proof uses the 
Keen-Halpern [64, 92] collar lemma. A different proof, and a numerical estimate for $\alpha_{p}$, was recently given by Buser [38].

(C) Now let $\Sigma^{(1)}, \Sigma^{(2)}, \ldots, \Sigma^{(r)}$ be all topologically distinct terminal surfaces of genus $p$. The inequality in (B) implies that there are compact sets $F^{(j)} \subset \mathfrak{D}\left(\Sigma^{(j)}\right)$ such that the images of $F^{(1)}, \ldots, F^{(r)}$ under the natural maps $\mathfrak{D}\left(\Sigma^{(j)}\right) \rightarrow \hat{X}_{p}$ cover $\hat{X}_{p}$. We conclude that $\hat{X}_{p}$ is compact.

That $X_{p}$ can be compactified by adding to it moduli of algebraic curves of genus $p$ with nodes, and that the resulting space $\hat{X}_{p}$ is a normal complex space has been established by methods of algebraic geometry. It is also known that $\hat{X}_{p}$ is projective algebraic (Mumford and others [40, 94, 95]); this result has not yet been obtained by methods described here.

(D) A different construction of $\hat{X}_{p}$ has been indicated by Earle and Marden [52] and by Abikoff [3]. These authors work with the standard embedding $T_{p} \subset B_{2}\left(L, G_{0}\right)$, cf. $\$ 6$, and introduce a new topology in the augmented Teichmüller space $\hat{T}_{p}$ consisting of $T_{p}$ and all its regular boundary points, cf. $\S 10$, (F). The modular group $\operatorname{Mod}_{p}$ acts continuously on all of $\hat{T}_{p}$ and $\hat{X}_{p}$ is obtained as $\hat{T}_{p} / \operatorname{Mod}_{p}$. ( $\hat{T}_{p}$ is not a manifold.)

(E) On the other hand, Masur [90] proved that $\hat{X}_{p}$ can be obtained by completing $X_{p}$ with respect to the metric induced in it by the Weil-Petersson metric in $T_{p}$, cf. $\$ 7.7$.

\section{APPENDIX}

\section{Some auxiliary results.}

(A) Teichmüller spaces of Riemann surfaces which cannot be compactified (as Riemann surfaces) by adding finitely many points are infinite dimensional. We shall summarize the theory of such spaces in $\S 19$. Here we state some results from the theory of quasiconformal mappings and from functional analysis which will be needed later, and which are of independent interest.

(B) The following theorem is due to Ahlfors and Beurling [36].

An increasing continuous function $x \mapsto f(x)$ with $f(-\infty)=-\infty$ and $f(+\infty)$ $=+\infty$ is the restriction to $\mathbf{R}$ of a quasiconformal self-mapping $F$ of $\mathbf{C}$ if and only if it is quasisymmetric, i.e., if and only if there is a constant $M>0$ such that

$$
\frac{1}{M} \leqslant \frac{f(x+y)-f(x)}{f(x)-f(x-y)} \leqslant M .
$$

Futhermore, $K(F)$ can be estimated in terms of $M$ and vice versa.

One may always assume that $F(\bar{z})=\overline{F(z)}$. Quasisymmetric functions are the restrictions to the real axis of continuous extensions of quasiconformal self-mappings of $U$ which fix $\infty$.

(C) A quasicircle $C$ is a Jordan curve in $\mathbf{C} \cup\{\infty\}$ which is the image of a circle under a quasiconformal self-mapping $\boldsymbol{F}$ of $\mathbf{C} \cup\{\infty\}$. The dilatation $K(C)$ is the infinum of $K(F)$ where $F$ runs over all such maps. 
Ahlfors [10] showed that a Jordan curve $C$ containing the point $\infty$ is a quasicircle if and only if there is a constant $M$ such that for any 3 finite points $a, b, c$ on $C$ with $b$ between $a$ and $c$,

$$
\left|\frac{b-a}{c-a}\right| \leqslant M
$$

and if and only if there is Lipschitz continuous quasireflection about $C$, i.e., a topological orientation reversing self-map $\Phi$ of $C$, such that $\Phi \circ \Phi=$ id, $\Phi \mid C=$ id and there is a constant $M^{\prime}$ such that

$$
\left|\Phi\left(z_{1}\right)-\Phi\left(z_{2}\right)\right| \leqslant M^{\prime}\left|z_{1}-z_{2}\right| \text {. }
$$

Furthermore $K(C)$ can be estimated in terms of $M$ (or of $M^{\prime}$ ) and vice versa.

(D) Let $D$ be a domain in $\mathbf{C} \cup\{\infty\}$ with at least two boundary points, $\lambda(z)|d z|$ the Poincaré metric in $D, \Gamma$ a discrete group of conformal self-mappings of $D$, which may be the trivial group $1=\{$ id $\}$, and $q>1$ an integer. An automorphic form of weight $(-2 q)$ for $\Gamma$ in $D$ is a holomorphic function $\varphi(z), z \in D$, such that $\varphi(z)=O\left(|z|^{-2 q}\right), z \rightarrow \infty$, if $\infty \in D$ and

$$
\varphi(\gamma(z)) \gamma^{\prime}(z)^{q}=\varphi(z) \text { for } \gamma \in \Gamma \text {. }
$$

The automorphic forms $\varphi$ with

$$
\|\varphi\|_{A}=\iint_{D / \Gamma} \lambda(z)^{2-q}|\varphi(z)| d x d y<\infty
$$

form the Banach space $A_{q}(D, \Gamma)$ of integrable forms, those with

$$
\|\varphi\|_{B}=\sup \left|\lambda(z)^{-q} \varphi(z)\right|<\infty
$$

form the Banach space $B_{q}(D, \Gamma)$ of bounded forms. The main properties of these spaces are as follows $[23,73]$.

There is a bounded linear map $\Theta$ of $A_{q}(D, 1)$ onto $A_{q}(D, \Gamma)$ obtained by forming the Poincaré theta series

$$
(\Theta \Phi)(z)=\sum_{\gamma \in \Gamma} \Phi(\gamma(z)) \gamma^{\prime}(z)^{q},
$$

and there is a pairing (Petersson's scalar product) between $A_{q}(D, \Gamma)$ and $B_{q}(D, \Gamma)$ given by

$$
(\varphi, \psi)_{D, \Gamma}=\iint_{D / \Gamma} \lambda(z)^{2-2 q} \varphi(z) \overline{\psi(z)} d x d y
$$

(for $\varphi \in A, \psi \in B$ ) which establishes a topological bijection between $B_{q}(D, \Gamma)$ and $A_{q}(D, \Gamma)^{*}$.

The spaces $A_{q}(D, \Gamma)$ and $B_{q}(D, \Gamma)$ are finite dimensional if and only if the group $\Gamma$ is finitely generated and the Fuchsian group $h \Gamma h^{-1}$ (where $h$ is a conformal map of the universal covering surface of $D$ onto $U$ ) has every point of $\mathbf{R}$ as a limit point.

If so, $A_{q}(D, \Gamma)=B_{q}(D, \Gamma)$.

(E) While the above statements have nothing to do with quasiconformal mappings the proof of the following theorem [24] depends on Ahlfors' characterization of quasicircles. 
Let $C$ be an oriented Jordan curve in $\hat{\mathbf{C}} \cup\{\infty\}, D_{1}$ and $D_{2}$ the domain interior and exterior to $C$, respectively, $\lambda_{j}(z)|d z|$ the Poincare metric in $D_{j}$, $j=1,2$, and $q>1$ an integer. Let $\Gamma$ be a discrete group of Möbius transformations with map $D_{1}$ (and $D_{2}$ ) onto itself. Set

$$
\left(\varrho_{C} \varphi\right)(z)=\iint_{D_{1}} \frac{\lambda_{1}(\zeta)^{2-2 q} \overline{\varphi(\zeta)} d \xi d \eta}{(\zeta-z)^{2 q}}, \quad z \in D,
$$

and let $\mathcal{L}_{-C}$ be defined similarly, with $D_{1}$ and $D_{2}$ interchanged. If $C$ is $a$ quasicircle, $\mathcal{L}_{C}$ is a topological bijection of $A_{q}\left(D_{1}, \Gamma\right)$ onto $A_{q}\left(D_{2}, \Gamma\right)$ and of $B_{q}\left(D_{1}, \Gamma\right)$ onto $B_{q}\left(D_{2}, \Gamma\right)$. Furthermore

$$
\left(\mathfrak{L}_{C} \varphi, \psi\right)_{D_{2}, \Gamma}=\left(\varphi, \mathfrak{L}_{-C} \psi\right)_{D_{1}, \Gamma}
$$

for $\varphi \in A_{q}\left(D_{1}, \Gamma\right)$ and $\psi \in B_{q}\left(D_{2}, \Gamma\right)$.

\section{Infinite dimensional Teichmüller spaces.}

(A) In the theory of finite dimensional Teichmüller spaces, summarized in $\S \S 2-13$, quasiconformal mappings are a tool for proving theorems. In the theory of infinite dimensional Teichmüller spaces [21, 22, 27] quasiconformal mappings necessarily appear in the basic definitions.

Let $S$ be an arbitrary Riemann surface (without nodes) which is not a sphere with 0,1 or 2 punctures and not a torus and let $f: S \rightarrow S_{0}$ be a quasiconformal bijection onto another such surface. Then $f$ extends to a topological bijection of $S \cup b(S)$, where $b(S)$ is the union of all ideal boundary curves of $S$, onto $S_{0} \cup b\left(S_{0}\right)$. We shall denote this extension by the same letter $f$.

Two quasiconformal maps, $f_{1}: S_{1} \rightarrow S$ and $f_{2}: S_{2} \rightarrow S$ and called weakly equivalent if there is a commutative diagram

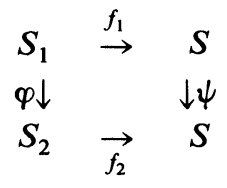

where $\varphi$ is conformal and $\psi$ homotopic to the identity. The maps $f_{1}$ and $f_{2}$ are called equivalent if $\psi$, or rather its continuous extension, can be continuously deformed into the identity leaving the image of every point in $b(S)$ fixed.

The equivalence classes

$$
[f]=\left[f: S^{\prime} \rightarrow S\right]
$$

of quasiconformal maps are the points of the Teichmüller space $T(S)$, the weak equivalence classes

$$
(f)=\left(f: S^{\prime} \rightarrow S\right)
$$

are the points of the reduced Teichmüller space $T^{\#}(S)$. There is an obvious canonical surjection

$$
T(S) \rightarrow T^{\#}(S)
$$

which is the identity if $b(S)=\varnothing$; cf. $2,(\mathrm{~A})$ and $(\mathrm{F})$. 
The Teichmüller distance between two points $\left[f_{1}\right]$ and $\left[f_{2}\right]$ in $T(S)$ is defined as

$$
\frac{1}{2} \log \inf K(f), \quad f \in\left[f_{1}^{-1} \circ f_{2}\right],
$$

where $K$ denotes the dilatation. The Teichmüller distance between two points in $T^{\#}(S)$ can be defined similarly, or by using the map (19.1). Both spaces $T(S)$ and $T^{\#}(S)$ are complete metric spaces.

Every quasiconformal bijection $\omega: S_{0} \rightarrow S_{1}$ induces allowable mappings

$$
\omega_{*}: T\left(S_{0}\right) \rightarrow T\left(S_{1}\right), \quad T^{\#}\left(S_{0}\right) \rightarrow T^{\#}\left(S_{1}\right)
$$

which take $[f]$ into $[\omega \circ f]$ and $(f)$ into $(\omega \circ f)$. These mappings are isometries.

An allowable self-mapping of $T(S)$ or of $T^{\#}(S)$ is called a modular transformation.

(B) It is clear that if $S$ is compact, except for $n$ punctures, and of genus $p$, then $T(S)$ can be identified with $T_{p, n}$, and if $S$ is compact except for $n$ punctures and $m$ "holes", and of genus $p$, then $T^{\#}(S)$ can be identified with $T_{p, n, m}^{\#}$. It turns out that in all other cases $T(S)$ and $T^{\#}(S)$ are infinite dimensional.

The theory of $T(S)$ is richer than that of $T^{\#}(S)$. In particular, $T(S)$ has a natural complex structure, whereas $T^{\#}(S)$ has only a real analytic structure; $T^{\#}(U)$ is a point, whereas $T(U)$ is a domain in an infinite dimensional complex Banach space (and is, in some sense, the "universal" Teichmüller space, see below). Also, the theory of $T^{\#}(S)$ can be reduced to that of $T(\hat{S})$ where $\hat{S}$ is obtained from $S$ by doubling, cf. $\S 2$, (F).

From now on we shall be concerned with $T(S)$. It is convenient to extend the definitions further.

(C) Let $Q$ denote the group of all conformal self-mappings of the upper half-plane $U$. Every $\omega \in Q$ extends to a homeomorphism of $U \cup \mathbf{R} \cup\{\infty\}$ which we denote by the same letter. We denote by $Q_{n}$ the subgroup of those $\omega \in Q$ which fix 0,1 and $\infty$, and by $Q_{0}$ the normal subgroup of those which leave every $x \in \mathbf{R}$ fixed. We may also consider $\operatorname{PSL}(2, \mathbf{R})$ as a subgroup of $Q$. If $G \subset P S L(2, \mathbf{R})$ is discrete, we denote by $Q(G)$ the set of those $\omega \in Q$ for which

$$
w G w^{-1} \subset P S L(2, \mathbf{R})
$$

and we set $Q_{n}(G)=Q(G) \cap Q_{n}$.

The Teichmüller space $T(G)$ is defined as the image of $Q_{n}(G)$ under the canonical map

$$
Q_{n} \rightarrow Q_{n} / Q_{0}
$$

If $\omega \in Q(G), \omega$ induces an allowable mapping $T(G) \rightarrow T\left(\omega^{-1} G \omega\right)$ which takes the point $[w] \in T(G)$ defined by $w \in Q_{n}(G)$ into $[\alpha \circ w \circ \omega]$ where $\alpha$ is an element of $\operatorname{PSL}(2, \mathbf{R})$ chosen so that $\alpha \circ w \circ \omega \in Q_{n}$. Allowable self-mappings of $T(G)$ are called modular transformations, they are induced by elements of the normalizer of $G$ in $Q$. 
(D) The group $Q_{n}$ is a complete metric space (but not a topological group) under the Teichmüller metric

$$
\langle w, \hat{w}\rangle=\frac{1}{2} \log K\left(\hat{w} \circ w^{-1}\right)
$$

(where $K$ is the dilatation). This metric defines a Teichmüller metric $\delta_{G}$ in $T(G)$ via the canonical mapping $Q_{n}(G) \rightarrow T(G)$.

The Teichmüller spaces $T(G)$ are complete and allowable mappings are isometries.

(E) For $G=1$ the space $T(G)=T(1)$ is the group $Q_{n} / Q_{0}$, that is, the group of orientation-preserving quasisymmetric (cf. $\S 18$, (B)) self-maps of $\mathbf{R}$ which fix 0 and 1.

By our definitions

$$
T(G) \subset T\left(G_{1}\right) \text { if } G_{1} \subset G .
$$

In particular, since $\{$ id $\}=1 \subset G$, we have that $T(G) \subset T(1)$ for all Fuchsian groups $G$. For this reason $T(1)$ is called the universal Teichmüller space.

On every $T(G)$ the Teichmüller metric $\delta_{1}$ and $\delta_{G}$ are topologically equivalent and $\delta_{1} \leqslant \delta_{G}$. Recently Strebel [111] showed that, in general, $\delta_{1}<\delta_{G}$.

(F) The connection between the Teichmüller spaces defined in $\$ 2$ and those defined above is given by an isomorphism theorem below.

For every Fuchsian group $G \subset P S L(2, \mathbf{R})$ let $U_{G}$ denote the complement in $U$ of the fixed points of elliptic elements of $G$, so that $U_{G} / G$ is a Riemann surface (and $U_{G}=U$ if $G$ is torsion free).

Every conformal bijection $h: U_{G} / G \rightarrow S$ induces a homeomorphism of $T(G)$ onto $T(S)$. These homeomorphisms respect Teichmüller distances and allowable mappings.

In particular, $T(U)$ may be identified with $T(1)$.

If $G$ has no torsion, the theorem follows directly from the definitions. Given $G, S=h(U / G)$ and $w \in Q_{n}(G)$, set $S^{\prime}=U /\left(w G w^{-1}\right)$ and let $F$ be the (quasiconformal) map which makes the diagram

$$
\begin{array}{cccc} 
& U & \stackrel{w}{\rightarrow} & U \\
& \downarrow & & \downarrow \\
S \stackrel{h}{\leftarrow} & U / G & \stackrel{F}{\rightarrow} & U /\left(w G w^{-1}\right)=S^{\prime}
\end{array}
$$

commutative (where vertical arrows denote canonical projections). Then the desired homeomorphism takes $[w] \in T(G)$ into $[f] \in T(S)$ where $f=$ $h \circ F^{-1}$. The original proofs of the isomorphism theorem for the general case (by Marden [82] and by Bers-Greenberg [34]) were rather complicated, but Kra [74] observed that a short proof can be based on Teichmüller's theorem.

REMARK. One can also define reduced Teichmüller spaces $T^{\#}(G)$ and prove for them a corresponding isomorphism theorem.

(G) We can now repeat the construction in $\$ 6$ and embed $T(G)$ into a Banach space, namely into the space $B_{2}(L, G)$ where $L$ is the lower halfplane. 
If $\tau=[w] \in T(G)$, there is a quasiconformal homeomorphism $\hat{w}$ of $\mathbf{C} \cup\{\infty\}$ onto itself such that $\hat{w} \circ w^{-1} \mid U$ and $\hat{w} \mid L$ are conformal, and $\hat{w}$ keeps $0,1, \infty$ fixed. The Schwarzian derivative $\varphi^{\tau}$ of $\hat{w} \mid L$ depends only on $\tau=[w]$ and not on the particular choice of $w$, and $\varphi^{\tau}$ lies in a ball of radius $\frac{3}{2}$ in $B_{2}(L, G)$. The mapping

$$
T(G) \ni \tau \mapsto \varphi^{\tau} \in B_{2}(L, G)
$$

is a homeomorphism onto a domain.

The proof of the latter statement is somewhat involved. It was first given by Ahlfors [10] for the case $G=1$. The author's proof [21] used Ahlfors' theorems stated in $\$ 18$, (C) to derive the theorem in $\$ 18$, (E) and from it the desired result, for all $G$. Later Earle [41] found a simple way to go from $G=1$ to an arbitrary $G$.

The embedding theorem gives a complex structure to $T(G)$ and, in view of the isomorphism theorem of $(\mathrm{F})$, also to $T(S)$. It turns out that all allowable mappings are holomorphic.

(H) We may identify $T(G)$ with its image in $B_{2}(L, G)$ under (19.1). It has been shown that $T(G)$ is holomorphically convex for every $G$.

Also

$$
T(G)=T(1) \cap B_{2}(L, G) .
$$

This is almost evident if $\operatorname{dim} T(G)<\infty$, due to $\mathrm{Kra}$ if $G$ is finitely generated, and has been recently proved by Tukia [119] in the general case.

(I) Some results in $\$ \S 9$ and 10 can be extended to the general case. We omit the details and refer the reader to the literature.

(J) We conclude by some remarks about the connection between the universal Teichmüller space $T(1)$ and univalent functions.

Let $\mathcal{S}$ denote the set of Schwarzian derivatives of univalent functions $f(z)$, $z \in L$. Then $\delta$ is a bounded closed set in $B=B_{2}(L, 1)$ and $T(1)$ may be described as the set of those $\varphi \in \mathcal{S}$ for which $f(L)$ is a quasidisc (i.e., a domain bounded by a quasicircle, cf. $\S 18,(\mathrm{C})$ ).

The boundary $\partial T(1)$ of $T(1)$ in $B$ lies in $\delta$. Gehring $[58,59]$ proved that $T(1)$ is the interior of $\mathcal{S}$, but that the complement of $T(1) \cup \partial T(1)$ in $\mathcal{S}$ is not empty.

It seems interesting to determine which simply connected domains $D$ have the property that the Schwarzian derivatives of the conformal maps $L \rightarrow D$ belong to $\partial T(1)$. It is also interesting to describe precisely the set $\partial \mathcal{S}-\partial T(1)$. At present we do not know whether $\mathcal{S} \subset B$ is connected.

\section{REFERENCES}

1. W. Abikoff, Some remarks on Kleinian groups, Advances in the Theory of Riemann Surfaces, Ann. of Math. Studies, No. 66, Princeton Univ. Press, Princeton, N. J., 1971, pp. 1-5.

2. On boundaries of Teichmüller spaces and on Kleinian groups. III, Acta Math. 134 (1975), 212-237.

3. ___ Augmented Teichmüller spaces, Bull. Amer. Math. Soc. 82 (1976), 333-334.

4. ___ Degenerating families of Riemann surfaces, Ann. of Math. (2) 105 (1977), 29-44. 
5. __ Topics in the real analytic theory of Teichmüller space, Lecture Notes in Math., vol. 820, Springer-Verlag, Berlin and New York, 1980.

6. L. V. Ahlfors, On quasiconformal mappings, J. d'Analyse Math. 3 (1953/54), 1-58.

7. The complex analytic structure of the space of closed Riemann surfaces, $\mathbf{R}$. Nevanlinna et al., Analytic Functions, Princeton Univ. Press, Princeton, N. J., 1960, pp. 45-66.

8. __ Curvature properties of Teichmüller's space, J. d'Analyse Math. 9 (1961), 161-176.

9. __ Some remarks on Teichmüller's space of Riemann surfaces, Ann. of Math. (2) 74 (1961), 171-191.

10. __ Quasiconformal reflections, Acta Math. 109 (1963), 291-301.

11. __ Finitely generated Kleinian groups, Amer. J. Math. 86 (1964), 413-423; 87 (1965), 759.

12. Fundamental polyhedrons and limit sets of Kleinian groups, Proc. Nat. Acad. Sci.

U.S.A. 55 (1966), 251-254.

13. __ Lectures on quasiconformal mappings, Van Nostrand, New York, 1966.

14. Quasiconformal mappings, Teichmüller spaces, and Kleinian groups, Proc. Internat. Congr. Math. (Helsinki 1978), pp. 71-84.

15. L. V. Ahlfors and L. Bers, Riemann's mapping theorem for variable metrics, Ann. of Math. (2) 72 (1960), 385-404.

16. W. L. Baily, Jr., On the theory of $\theta$-functions, the moduli of abelian varieties, and the moduli of curves, Ann. of Math. (2) 75 (1962), 342-381.

17. L. Bers, Quasiconformal mappings and Teichmüller's theorem, R. Nevanlinna et. al., Analytic Functions, Princeton Univ. Press, Princeton, N. J., 1960, pp. 89-119.

18. __ Simultaneous uniformization, Bull. Amer. Math. Soc. 66 (1960), 94-97.

19. Correction to Spaces of Riemann surfaces as bounded domains, Bull. Amer. Math. Soc. 67 (1961), 465-466.

20. Uniformization by Beltrami equations, Comm. Pure. Appl. Math. 14 (1961), 215-228.

21. On moduli of Riemann surfaces, Lecture notes, E.T.H., Zurich, 1964.

22. __ Automorphic forms and general Teichmüller spaces, Proc. Conf. Complex Analysis (Minneapolis, 1964) pp. 109-113, Springer-Verlag, Berlin and New York, 1965.

23. Automorphic forms and Poincaré series for infinitely generated Fuchsian groups, Amer. J. Math. 87 (1965), 196-214.

24. , A non-standard integral equation with applications to quasiconformal mappings, Acta Math. 116 (1966), 113-134.

25. _ On boundaries of Teichmüller spaces and on Kleinian groups. I, Ann. of Math. (2) 91 (1970), 570-600.

26. Uniformization, moduli and Kleinian groups, Bull. London Math. Soc. 4 (1972), 257-300.

27. __ Fiber spaces over Teichmüller spaces, Acta Math. 130 (1973), 89-126.

28. __ On spaces of Riemann surfaces with nodes, Bull. Amer. Math. Soc. 80 (1974), 1219-1222.

29. Deformations and moduli of Riemann surfaces with nodes and signatures, Math. Scand. 36 (1975), 12-16.

30. __ Quasiconformal mappings, with applications to differential equations, function theory and topology, Bull. Amer. Math. Soc. 83 (1977), 1083-1100.

31. An extremal problem for quasiconformal mappings and a theorem by Thurston, Acta Math. 141 (1978), 73-98.

32. The action of the modular group on the complex boundary, Riemann Surfaces and Related Topics: Proceedings of the 1978 Stony Brook Conference, Ann. of Math. Studies, No. 97 1981, pp. 33-52.

33. L. Bers and L. Ehrenpreis, Holomorphic convexity of Teichmüller spaces, Bull. Amer. Math. Soc. 70 (1964), 761-764.

34. L. Bers and L. Greenberg, Isomorphisms between Teichmüller spaces, Advances in the theory of Riemann surfaces, Ann. of Math. Studies, No. 66, 1971, pp. 53-79.

35. L. Bers and I. Kra, (eds.), A crash course on Kleinian groups, Lecture Notes in Math., vol. 400, Springer-Verlag, Berlin and New York, 1974. 
36. A. Beurling and L. V. Ahlfors, The boundary correspondence under quasiconformal mappings, Acta Math. 96 (1956), 125-142.

37. R. Bowen, Hausdorff dimension of quasicircles, Inst. Hautes Études Sci. Publ. Math. 50 (1979), 153-170.

38. P. Buser, Riemannsche Flächen und Längenspektrum vom trigonometrischen Standpunkt aus Habilitationsschrift, Universität Bonn, 1980.

39. T. Chu, The Weil-Petersson metric in the moduli space, Chinese J. Math. 4 (1976), 29-51.

40. P. Deligne and D. Mumford, The irreducibility of the space of curves of a given genus, Inst. Hautes Etudes Sci. Publ. Math. 36 (1969), 75-109.

41. C. J. Earle, The Teichmüller space of an arbitrary Fuchsian group, Bull. Amer. Math. Soc. 70 (1964), 699-701.

42. __ Teichmüller spaces of groups of the second kind, Acta Math. 112 (1964), 91-97.

43. ___ Reduced Teichmüller spaces, Trans. Amer. Math. Soc. 126 (1967), 54-63.

44. __ On holomorphic families of pointed Riemann surfaces, Bull. Amer. Math. Soc. 79 (1973), 163-166.

45. On the Carathéodory metric in Teichmüller spaces, Discontinuous Groups and Riemann Surfaces, Ann. of Math. Studies, No. 79, Princeton Univ. Press, Princeton, N. J., 1974, pp. 99-103.

46. __ The Teichmüller distance is differentiable, Duke Math. J. 44 (1977), 389-397.

47. __ Families of Riemann surfaces and their Jacobi varieties, Ann. of Math. 107 (1978), $255-286$.

48. C. J. Earle and J. Eells, On the differential geometry of Teichmüller spaces, J. Analyse Math. 19 (1967), 35-52.

49. , A fibre bundle description of Teichmüller theory, J. Differential Geom. 3 (1969), 19-43.

50. C. J. Earle and I. Kra, On holomorphic mappings between Teichmüller spaces, Contributions to Analysis, Academic Press, New York, 1974, pp. 107-124.

51. __ On sections of some holomorphic families of closed Riemann surfaces, Acta Math. 137 (1976), 49-79.

52. C. J. Earle and A. Marden (to appear).

53. M. Engber, Teichmüller spaces and representability of functors, Trans. Amer. Math. Soc. 201 (1975), 213-226.

54. A. Fathi, F. Laudenbach and V. Poénaru, Travaux de Thurston sur les surfaces, Astérisque 66-67 (1979), 1-284.

55. L. Ford, Automorphic functions (second ed.), Chelsea, New York, 1951.

56. R. Fricke and F. Klein, Vorlesungen über die Theorie der automorphen Funktionen (two volumes), B. G. Teubner, 1889 and 1926.

57. F. P. Gardiner, On relative Teichmüller spaces and a globalization principle in Riemann surface theory, J. d'Analyse Math. 35 (1979), 1-12.

58. F. W. Gehring, Univalent functions and the Schwarzian derivative, Comment. Math. Helv. 52 (1977), 561-572.

59. ___ Spirals and the universal Teichmüller space, Acta Math. 141 (1978), 99-113.

60. J. Gilman, On the Nielsen type and the classification for the mapping-class group, Advances in Math. 40 (1981), 68-96.

61. L. Greenberg, Fundamental polyhedra for Kleinian groups, Ann. of Math. 84 (1966), 433-441.

62. A. Grothendieck, Techniques de construction en géometrie analytique, Sém. Cartan (1961/62) exp. 17, (with an appendix by J. P. Serre).

63. K. T. Hahn, On the completeness of the Bergman metric and its subordinate metrics, Proc. Nat. Acad. Sci. U.S.A. 73 (1976), 42-44.

64. N. Halpern, Some contributions to the theory of Riemann surfaces, Thesis, Columbia University, 1978.

65. R. S. Hamilton, Extremal quasiconformal mappings with prescribed boundary values, Trans. Amer. Math. Soc. 138 (1969), 399-406.

66. W. J. Harvey, (ed.), Discrete groups and automorphic functions, Academic Press, New York, 1977. 
67. J. H. Hubbard, Sur les sections analytique de la courbe universelle de Teichmüller, Mem. Amer. Math. Soc. No. 166, 1976, pp. 1-137.

68. J. Hubbard and H. Masur, Quadratic differentials and foliations, Acta Math. 142 (1979), 221-274.

69. J. A. Jenkins, On the existence of certain general extremal metrics, Ann. of Math. 66 (1957), 440-453.

70. L. Keen, On Fricke moduli, Advances in the Theory of Riemann Surfaces, Ann. of Math. Studies, No. 66, 1971, pp. 205-224.

71. S. P. Kerckhoff, The asymptotic geometry of Teichmüller space, Topology 19 (1980), 23-41.

72. S. Kobayashi, Hyperbolic manifolds and holomorphic mappings, M. Dekker, New York, 1970.

73. I. Kra, Automorphic forms and Kleinian groups, Benjamin, New York, 1972.

74. ___ On new kinds of Teichmüller spaces, Israel J. Math. 16 (1973), 237-257.

75. _ On the Nielsen-Thurston-Bers type of some self-maps of Riemann surfaces, Acta Math. 146 (1981), 231-270.

76. I. Kra and B. Maskit, Involutions on Kleinian groups, Bull. Amer. Math. Soc. 78 (1972), 801-805.

77. W. Kraus, Über den Zusammenhang einiger Charakteristiken eines eintach zusammehangenden Bereiches mit der Kreisabbildung, Mitt. Math. Sem. Giessen 21 (1932), 1-28.

78. S. Kravetz, On the geometry of Teichmüller spaces and the structure of their modular groups, Ann. Acad. Sci. Fenn. 278 (1959), 1-35.

79. S. L. Krushkal, On Teichmüller's theorem on extremal quasiconformal mappings, Mat. Sb. 8 (1967), 313-332.(in Russian)

80. __ Quasiconformal mappings and Riemann surfaces, V. H. Winston and Sons, 1979.

81. O. Lehto and K. I. Virtanan, Quasiconformal mappings in the plane, Springer-Verlag, Berlin, 1973.

82. A. Marden, On homotopic mappings of Riemann surfaces, Ann. of Math. 90 (1969), 1-8.

83. B. Maskit, On Klein's combination theorem. II, Trans. Amer. Math. Soc. 131 (1968), 32-39.

84. __ On boundaries of Teichmüller spaces and on Kleinian groups. II, Ann. of Math. 91 (1970), 607-639.

85. On On Klein's combination theorem. III, Advances in the Theory of Riemann Surfaces, Ann. of Math. Studies, No. 66, 1971, pp. 297-316.

86. __ Moduli of marked Riemann surfaces, Bull. Amer. Math. Soc. 80 (1974), 773-777.

87. $249-270$

88. _ On the classification of Kleinian groups. II. Signatures, Acta. Math. 138 (1977), $17-42$.

89. H. Masur, On a class of geodesics in Teichmüller space, Ann. of Math. 102 (1975), 205-221.

90. The extension of the Weil-Petersson metric to the boundary of Teichmüller spaces, Duke Math. J. 43 (1976), 623-635.

91. The Jenkins-Strebel differentials with one cylinder are dense, Comment. Math. Helv. 54 (1979), 179-184.

92. J. P. Matelski, A compactness theorem for Fuchsian groups of the second kind, Duke Math. J. 43 (1976), 829-840.

93. R. T. Miller, Nielsen's view point on geodesic laminations, Advances in Math. (to appear).

94. D. Mumford, The structure of the moduli spaces of curves and abelian varieties, Proc. Intern. Congr. Math. (Nice 1970), vol. 1, pp. 457-465.

95. __ Curves and their Jacobians, Univ. of Michigan Press, 1975.

96. Z. Nehari, Schwarzian derivatives and schlicht functions, Bull. Amer. Math. Soc. 55 (1949), 545-551.

97. B. O'Byrne, On Finsler geometry and applications to Teichmüller spaces, Advances in the Theory of Riemann surfaces, Ann. of Math. Studies, No. 66, 1971, pp. 317-328.

98. D. B. Patterson, The Teichmüller spaces are distinct, Proc. Amer. Math. Soc. 35 (1972), $179-182 ; 38$ (1973), 668. 
99. R. M. Porter, Computation of a boundary point of Teichmüller space, Bol. Soc. Mat. Mexicana 24 (1979), 15-26.

100. H. E. Rauch, On the transcendental moduli of algebraic Riemann surfaces, Proc. Nat. Acad. Sci. U.S.A. 41 (1955), $42-49$.

101. _ A transcendental view of the space of algebraic Riemann surfaces, Bull. Amer. Math. Soc. 71 (1965), 1-39.

102. E. Reich and K. Strebel, Extremal quasiconformal mappings with given boundary values, Bull. Amer. Math. Soc. 79 (1973), 488-490.

103. , Extremal quasiconformal mappings with given boundary values, Contributions to Analysis, Academic Press, New York, 1974, pp. 375-391.

104. H. L. Royden, Report on the Teichmüller metric, Proc. Nat. Acad. Sci. U.S.A. 65 (1970), 497-499.

105. , Automorphisms and isometries of Teichmüller space, Advances in the Theory of Rieman Surfaces, Ann. of Math. Studies, No. 66, 1971, pp. 369-383.

106. M. Schiffer, A variational method for univalent quasi-conformal mappings, Duke Math. J. 33 (1966), 395-411.

107. K. Strebel, Zur Frage der Eindeutigkeit extremaler quasikonformer Abbildungen des Einheitskreises, Comment. Math. Helv. 36 (1961-62), 306-343; 39 (1964), 77-89.

108. __ Uber quadratische Differentiale mit geschlossenen Trajektorien und extremale quasikonforme Abbildungen, Festband zum 70 Geburtstag von Rolf Nevanlinna, Springer, Berlin, 1966, pp. 105-127.

109. __ On quadratic differential and extremal quasiconformal mappings, Proc. Int. Cong. Math. (Vancouver, 1974), pp. 223-227.

110. On On Acad. Sci. Fennicae 2 (1976), 533-551.

111. , On lifts of extremal quasiconformal mappings, J. d'Analyse Math. 31 (1977), 191-203.

112. On quasiconformal mappings of open Riemann surfaces, Comment. Math. Helv. 53 (1978), 301-321.

113. D. Sullivan, On the ergodic theory of infinity of an arbitrary discrete group of hyperbolic motions, Riemann Surfaces and Related Topics: Proceedings of the 1978 Stony Brook Conference, Ann. of Math. Studies, No. 97, 1980, pp. 465-496.

114. O. Teichmüller, Extremale quasikonforme Abbildungen und quadratische Differentiale, Preuss. Akad. 22 (1939).

115. , Bestimmung der extremalen quasi konformen Abbildungen bei geschlossenen orientierten Riemannschen Flächen, Preuss Akad. 4 (1943).

116. __ Veränderliche Riemannsche Flächen Deutsche Mathematik 7 (1949), 344-359.

117. W. P. Thurston, On the geometry and dynamics of diffeomorphisms of surfaces. I, preprint. 118. The geometry and topology of three-manifolds, Lecture Notes, Princeton University.

119. P. Tukia, Quasiconformal extensions of quasisymmetric mappings compatible with a Fuchsian group (to appear).

120. A. Weil, Sur les modules des surfaces de Riemann, Sém. Bourbaki, 1958.

121. S. Wolpert, Noncompleteness of the Weil-Petersson metric for Teichmüller space, Pacific J. Math. 61 (1975), 573-577.

Department of Mathematics, Columbia University, New York, New York 10027 